\title{
Quark-model calculation of charmed-baryon production by neutrinos
}

\author{
C. Avilez, ${ }^{+\dagger}$ T. Kobayashi, ${ }^{* \ddagger}$ and J. G. Körner \\ Deutsches Elektronen-Synchrotron DESY, Hamburg, West Germany
}

(Received 13 December 1976)

\begin{abstract}
We study the neutrino production of 25 low-lying charmed-baryon resonances in the four-flavor quark model. The mass difference of ordinary and charmed quarks is explicitly taken into account. The quark model is used to determine the spectrum of the charmed-baryon resonances and the $q^{2}=0$ values of the weak-current transition matrix elements. These transition matrix elements are then continued to spacelike $q^{2}$ values by a generalized meson-dominance ansatz for a set of suitably chosen invariant form factors. We find that the production of the $L=0$ states $C_{0}, C_{1}$, and $C^{*}{ }_{1}$ is dominant, with the $C_{0}$ produced most copiously. For $L=1,2$ the $J^{P}=3 / 2^{-}, 5 / 2^{+}$charm states are dominant. We discuss the sensitivity of our results to various input assumptions, e.g., the ratio of charm-quark mass to ordinary-quark mass. We give differential cross sections, total cross sections, and energy-integrated total cross sections using experimental neutrino fluxes. We conclude that the charmed-baryon production rate can be as large as $3-4 \%$ of the total neutrino cross section in a region extending from charm threshold to $E \simeq 10 \mathrm{GeV}$.
\end{abstract}

\section{INTRODUCTION}

Recently a charmed-baryon candidate with a mass of $2.26 \mathrm{GeV}$ was found in a photoproduction experiment at Fermilab. ${ }^{1}$ Some possible indirect evidence for charmed-baryon production was also reported on in neutrino interáctions by the observation of dilepton events (see e.g., Ref. 2) and $\Delta S=-\Delta Q$ events. $^{3}$ Together with the identification of charmed mesons in $e^{+} e^{-}$production, ${ }^{4}$ these results have given strong support to the charm interpretation of the new phenomena observed in highenergy physics since the discovery of the $J(\psi)$ particle in 1974.

Lee and Shrock (LS) have already shown that the quasielastic neutrino production of charmed baryon may constitute a sizable fraction of the total neutrino cross section $(\simeq 3 \%$ at $E=10 \mathrm{GeV})$ close to the charmed-baryon production thresholds ${ }^{5}$ even though the form of the GIM current ${ }^{6}$ disfavors these by a $\sin ^{2} \theta_{C}$ suppression factor. At higher energies as more and more charm channels open up, one expects the charm-production cross section to increase linearly with energy including contributions proportional to $\cos ^{2} \theta_{C}$ coming from the excitation of sea quarks in the low- $x$ region. Since the quasielastic charmed-baryon production cross sections are expected to level off at higher energies, the contribution of these channels will become less important. However, in an intermediate-energy region one may hope to collect enough data to be able to study the dynamical mechanisms leading to weak excitation of charmed-baryon resonances.

Even though there exists now strong evidence that charmed particles fill up the remaining slots of SU(4) multiplets confirming the most basic prediction of SU(4) symmetry, the large mass splittings between charmed and noncharmed SU(4)- multiplet members make it clear that the symmetry is badly broken. In the quark model SU(4) breaking basically manifests itself in large quark mass differences. Within the context of the quark model the neutrino production of charmed baryons affords a very nice way to study such a basic SU(4)-breaking mechanism, since the neutrino current induces a transition from a light noncharmed to a heavy charmed quark.

In the framework of the quark model we shall study the neutrino excitation of 25 charmed-baryon states with $C=1, S=0$ in the lowest radial mode with the orbital excitations $L=0,1$, and 2 (see Ref. 7). SU(4)-breaking effects are incorporated at the quark level by introducing quark mass breaking. We shall be using quark-model results only at $q^{2}=0$, where the quark model has been quite successful in accounting for the photoexcitation feature of the low-lying baryon resonances. For $q^{2} \neq 0$ the quark-model results become increasingly frame- and model-dependent and tend to be unreliable. ${ }^{8}$ Nor do the helicity amplitudes calculated in the quark model in general exhibit the correct structure of relativistic kinematics. We therefore calculate the values of the helicity amplitudes at general spacelike $q^{2}$ by continuing instead in terms of invariant form factors via a generalized-meson-dominance (GMD) $q^{2}$-dependence. Since the mesons that determine the formfactor behavior have charm $C=1$, the charm mass scale appropriate for charm-particle production is included in our form factors. As a technical aside we give a systematic discussion of the large$q^{2}$ behavior of the invariant form factors necessary to guarantee that production cross sections of higher-spin particles do not become spuriously large because of momentum-factor enhancements. Our paper is planned as follows. In Sec. II we 
discuss the quark-model classification of the lowlying $C=1, S=0$ charmed baryon states. Since their mass values are needed in the later crosssection calculations we also present mass estimates. In Sec. III we set up the kinematics of baryon-resonance production including a discussion of the large $-q^{2}$ behavior of transition form factors. As mentioned before, one of the main dynamical ingredients of our calculation is the use of the quark model to obtain the $q^{2}=0$ values of the weak-current transition form factors. This is done in Sec. IV, where we also discuss how the quark mass difference enters into this evaluation. In Sec. IV we also discuss how the transition form factors are continued to general $q^{2}$ using GMD. Our numerical results are presented in Sec. V. We give differential cross sections, total cross sections, and energy-integrated total cross sections using experimental neutrino fluxes. We discuss the dependence of our results on (i) the input charmed-baryon mass values, (ii) the ratio [c-quark mass $] /[(\mathbb{P}, \mathfrak{Y})$-quark mass $]$, and (iii) the mass values of the $C=1$ mesons in the GMD form factors. We also compare our calculation to two recent evaluations of the production of $L=0$ ground-state charmed baryons. Our results are summarized in Sec. VI. The more detailed aspects of our evaluation have been collected in Appendixes A-C.

\section{SPECTRUM OF CHARMED BARYONS}

One can easily obtain the spectrum of charmed baryons from the ordinary baryons with strangeness $S=-1\left(\Lambda, \Sigma, Y_{1}^{*}, \ldots\right)$ by replacing a $\lambda$ quark with a $c$ quark. The charmed baryons with $C=1$, $S=0$ can then be seen to belong to the $\underline{1}, \underline{8}$, and 10 representations of the subgroup $\mathrm{SU}(3)_{\text {Prc }}$ of $\mathrm{SU}(\overline{4)}$, which has $\mathbb{P}, \Re$, and $c$ as a basis representation just as the $C=0, S=1$ baryons are members of the 1,8 , and 10 representations of the ordinary $\overline{S U}(\overline{3})_{\text {or } \lambda} \lambda_{\text {. }}$ The $L=0$ ground-state charmed baryons which belong to the SU(8)-symmetric state are described by

$$
\begin{aligned}
& C_{0} \equiv\left(s ; 8 ; 0 ; \frac{1}{2}^{+}\right), \\
& C_{1} \equiv\left(s ; 8 ; 1 ; \frac{1}{2}^{+}\right), \\
& C_{1}^{*} \equiv\left(s ; 10 ; 1 ; \frac{3}{2}^{+}\right),
\end{aligned}
$$

with the notation (symmetry of $\mathrm{SU}(8)$ state; representation of charmed SU(3) metric $(m=$ mixed symmetric).

The first excited charmed baryons with orbital momentum $L=1$ belong to a mixed-symmetric state of $\mathrm{SU}(8)$, i.e.,

$$
\begin{aligned}
& B_{0}^{1} \equiv\left(m ; 8 ; 0 ; \frac{1}{2}^{-}\right), \quad B_{0}^{3} \equiv\left(m ; 8 ; 0 ; \frac{3}{2}^{-}\right), \\
& B_{1}^{1} \equiv\left(m ; 8 ; 1 ; \frac{1}{2}^{-}\right), \quad B_{1}^{3} \equiv\left(m ; 8 ; 1 ; \frac{3}{2}^{-}\right) \text {, } \\
& B_{0}^{* 1} \equiv\left(m ; 1 ; 0 ; \frac{1}{2}^{-}\right), \quad B_{0}^{* 3} \equiv\left(m ; 1 ; 0 ; \frac{3}{2}^{-}\right), \\
& B_{1}^{*^{1}} \equiv\left(m ; 10 ; 1 ; \frac{1}{2}^{-}\right), \quad B_{1}^{*^{3}} \equiv\left(m ; 10 ; 1 ; \frac{3}{2}^{-}\right) \text {， } \\
& B_{0}^{\prime 1} \equiv\left(m ; 8 ; 0 ; \frac{1}{2}^{-}\right), \quad B_{0}^{\prime^{3}} \equiv\left(m ; 8 ; 0 ; \frac{3}{2}^{-}\right), \\
& B_{0}^{\prime 5} \equiv\left(m ; 8 ; 0 ; \frac{5}{2}^{-}\right), \quad B_{1}^{\prime 1} \equiv\left(m ; 8 ; 1 ; \frac{1}{2}^{-}\right), \\
& B_{1}^{\prime 3} \equiv\left(m ; 8 ; 1 ; \frac{3}{2}^{-}\right), \quad B_{1}^{\prime 5} \equiv\left(m ; 8 ; 1 ; \frac{5}{2}^{-}\right),
\end{aligned}
$$

where $B_{A}^{B}=B_{I}^{2 J}$ and $B_{A}^{* B}$ denotes either the isosinglet of the 1 representation or the isotriplet member of the 10 representation. All these states have total quark spin $\frac{1}{2}$. The other six states $B_{A}^{\prime B}$ transforming as $\frac{8}{6}$ have total quark spin $\frac{3}{2}$. In the case of the $L=2$ excited charmed baryons one can have symmetric or mixed-symmetric $\mathrm{SU}(8)$ states. Since there are no indications yet for the existence of mixed-symmetric states with $L=2$ in the case of the ordinary baryon, i.e., for the $N^{*}, \Lambda^{*}$, $\Sigma^{*}$, and $\Xi^{*}$ states, we shall discuss only the symmetric states, i.e.,

$$
\begin{aligned}
& A_{0}^{3} \equiv\left(s ; 8 ; 0 ; \frac{3}{2}^{+}\right), \quad A_{0}^{5} \equiv\left(s ; 8 ; 0 ; \frac{5}{2}^{+}\right), \\
& A_{1}^{3} \equiv\left(s ; 8 ; 1 ; \frac{3}{2}^{+}\right), \quad A_{1}^{5} \equiv\left(s ; 8 ; 1 ; \frac{5}{2}^{+}\right), \\
& A_{1}^{*^{1}} \equiv\left(s ; 10 ; 1 ; \frac{1}{2}^{+}\right), \quad A_{1}^{*^{3}} \equiv\left(s ; 10 ; 1 ; \frac{3}{2}^{+}\right), \\
& A_{1}^{*^{5}} \equiv\left(s ; 10 ; 1 ; \frac{5}{2}^{+}\right), \quad A_{1}^{*^{7}} \equiv\left(s ; 10 ; 1 ; \frac{7}{2}^{+}\right) .
\end{aligned}
$$

In Fig. 1 we show the $\underline{4}, \underline{20}, \underline{20}$, and $\underline{4}$ * representations of SU(4), where we have accented the above $\mathrm{SU}(3)_{\text {orsc }}$ representations $\underline{3}, \underline{8}, \underline{10}$, and $\underline{1}$ with heavy lines and a heavy dot, respectively. As for the higher- $L$-excited states one can easily write down the different spin states in an analogous fashion.

Recently the observation of a charmed baryon was reported in a photoproduction experiment. ${ }^{1}$ The mass of the charmed baryon was given as $2.26 \pm 0.01 \mathrm{GeV}$. Following Ref. 9 we identify this charmed-baryon state with the $C_{0}^{+}$. Since at present there is no definite information on the mass values of heavier charmed-baryon states, we propose to evaluate the charmed-baryon mass spectrum in the following heuristic way.

$$
M_{C}=M_{Y}+\Delta_{c \lambda},
$$

where $M_{C}$ is the mass of the charmed-baryon counterpart of the strange baryon with mass $M_{\boldsymbol{Y}}$ and $\Delta_{c} \lambda$ is a measure of the mass difference of the $c$ and $\lambda$ quarks, which we propose to evaluate in the following way:

$$
\Delta_{c \lambda}=M_{C_{0}^{+}}-M_{\Lambda} \simeq 2.26-1.12=1.14 \mathrm{GeV}
$$

Based on Eqs. (2.4) and (2.5) we give the results of calculating the mass spectrum of the charmed baryons in Table I, where reference masses $M_{Y}$ are also given. ${ }^{10}$ It is interesting to note that, taking 


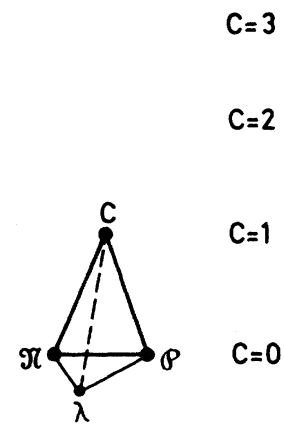

(a) 4

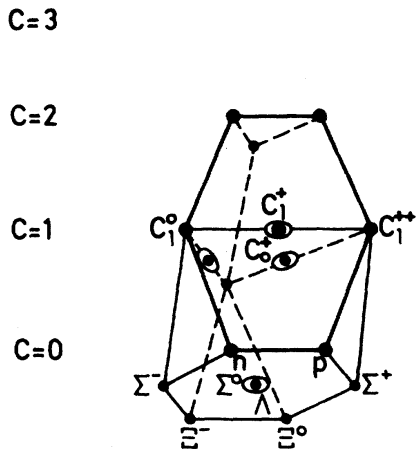

(b) $\underline{20}_{M}$

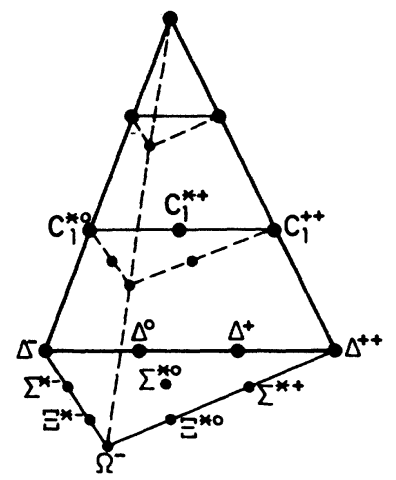

(c) $20 \mathrm{~s}$

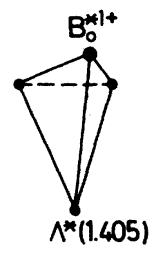

(d) $\underline{4}^{*}$

FIG. 1. SU(4) representations $\underline{4}, \underline{20} M, \underline{20} s$, and $\underline{4}^{*}$. Heavy lines and a heavy dot denote the SU $(3)_{\odot} \Re_{c}$ representations $\underline{3}, \underline{8}, \underline{10}$, and $\underline{1}$, respectively. The three-dimensional objects are viewed from below.

the masses of the charmed $J^{P}=\frac{1}{2}^{+}$state $\left[C_{0}^{+}(2.26)\right]$ and its recurrence at $J^{P}=\frac{5}{2}^{+}\left[A_{0}^{5}(2.95)\right]$, we obtain for the Regge slope

$$
\alpha_{C}^{\prime} \simeq 0.54 \mathrm{GeV}^{-2} \text {, }
$$

which is about one-half the universal slope and similar to the value assumed for the $D^{*}$ trajectory in our previous paper. ${ }^{7}$

De Rújula, Georgi, and Glashow ${ }^{11}$ have also given predictions for the masses of the ground-state $C=1, S=0$ charmed baryons. Using $M_{C_{0}}=2.26 \mathrm{GeV}$, one obtains from their predicted mass differences $M_{C_{1}}=2.42 \mathrm{GeV}$ and $M_{C_{1}^{*}}=2.48 \mathrm{GeV}$. These masses are $0.09 \mathrm{GeV}$ higher and $0.05 \mathrm{GeV}$ lower, respectively, than our mass values. It will be shown in Sec. IV that the calculated production cross sections are insensitive to mass variations on this scale. Finkelstein and Tuan ${ }^{12}$ predict mass values for the excited states $A_{0}^{5}, A_{2}^{5}$, and $A_{1}^{*^{7}}$ by using a universal Regge slope $\alpha^{\prime}=0.93 \mathrm{GeV}^{-2}$. They obtain the following mass values: $M_{A_{0}^{5}}=2.69, M_{A_{1}^{5}}=2.84$, and $M_{A_{1}^{* 7}}=2.90 \mathrm{GeV}$, where we obtain 2.96, 3.06, and $3.17 \mathrm{GeV}$, respectively. Again our cross-section results are not very much affected by changing the charmed-baryon masses on this scale.

\section{CROSS SECTION}

The neutrino production of charmed baryons and charmed-baryon resonances is repres ented by the process $\nu N \rightarrow \mu^{-} C$ in close analogy to the treatment of the neutrino production of ordinary baryon states in Refs. 13 and 14. Using the narrow-resonance approximation and neglecting the lepton mass one obtains a simple expression for the differential cross section $d \sigma / d q^{2}$ in terms of isobar rest-frame helicity amplitudes. ${ }^{14}$ One has

$$
\begin{aligned}
& \frac{d \sigma}{d q^{2}}=\frac{G_{c}^{2}}{4 \pi}\left[2 u v\left|f_{0}\right|^{2}+\frac{-q^{2}}{q_{c}{ }^{2}}\left(u^{2}\left|f_{+}\right|^{2}+v^{2}\left|f_{-}\right|^{2}\right)\right], \\
& 2 M^{2}\left|f_{ \pm}\right|^{2}=\frac{1}{2} \sum_{\lambda}\left|\left\langle B^{*}, \lambda^{*}=\lambda \pm 1\left|J_{ \pm}\right| B, \lambda\right\rangle\right|^{2}, \\
& 4 M^{2}\left|f_{0}\right|^{2}=\frac{1}{2} \sum_{\lambda}\left|\left\langle B^{*}, \lambda^{*}=\lambda\left|J_{0}+\frac{q_{0 c}}{q_{c}} J_{3}\right| B, \lambda\right\rangle\right|^{2},
\end{aligned}
$$

where $J_{ \pm}=\frac{1}{2}\left(J_{x} \pm i J_{y}\right)$, and where $\lambda$ and $\lambda^{*}$ are the helicities of the initial and final baryon of mass $m$ and $M$, respectively. The weak hadron current contains a vector and an axial-vector part $J_{\mu}=J_{\mu}^{V}+J_{\mu}^{A}$. $q_{0 c}$ and $q_{c}$ are the zero component and magnitude of three-momentum of the four-momentum transfer $q$ in the isobar rest system ( $q^{2}$ negative in the spacelike region)

$$
\begin{aligned}
& q_{c}=\frac{1}{2 M}\left\{\left[(M+m)^{2}-q^{2}\right]\left[(M-m)^{2}-q^{2}\right]\right\}^{1 / 2}, \\
& q_{0 c}=\frac{1}{2 M}\left(M^{2}-m^{2}+q^{2}\right) .
\end{aligned}
$$

The $u$ and $v$ are functions of $q^{2}$ and the lab energies $E$ and $E^{\prime}$ of the initial and final lepton:

$$
\begin{aligned}
& u=\frac{1}{2 E}\left(E+E^{\prime}+\frac{M}{m} q_{c}\right), \\
& v=\frac{1}{2 E}\left(E+E^{\prime}-\frac{M}{m} q_{c}\right) .
\end{aligned}
$$

Finally, $G_{C}{ }^{2}$ gives the strength of the weak-interaction coupling, where for the charm-production processes treated in this paper $G_{C}=G \sin \theta_{C}$ with $\sin \theta_{C}=0.235 \pm 0.005$.

The three contributions in Eq. (3.1) arising from $\left|f_{0}\right|^{2},\left|f_{+}\right|^{2}$, and $\left|f_{-}\right|^{2}$ are commonly referred to as the scalar $(S)$, left-handed $(L)$, and right-handed $(R)$ current contributions, respectively. The scalar contribution $\left|f_{0}\right|^{2}$ and the sum of left- and right- 
TABLE I. Masses of charmed baryons. The total quark spin is denoted by $S_{q}$. There are no $C=0, S=-1$ candidates for $B_{1}^{\prime 3}$ and $A_{1}^{* 1}$. Therefore we extrapolated the corresponding charmed-baryon mass value from the masses of $B_{1}^{\prime 1}$ and $B_{1}^{\prime 5}$ and $A_{1}^{*^{3}}, A_{1}^{* 5}$, and $A_{1}^{* 7}$, respectively.

\begin{tabular}{|c|c|c|c|c|c|c|}
\hline Particles & $J^{P}$ & $L$ & $S_{q}$ & $I$ & $\begin{array}{c}\text { Masses } \\
(\mathrm{GeV})\end{array}$ & $\begin{array}{c}\text { Reference } \\
\text { masses }\end{array}$ \\
\hline$c_{0}$ & $\frac{1}{2}^{+}$ & 0 & $\frac{1}{2}$ & 0 & 2.26 & $1.116(\Lambda)$ \\
\hline$c_{1}$ & $\frac{1}{2}^{+}$ & 0 & $\frac{1}{2}$ & 1 & 2.33 & $1.189(\Sigma)$ \\
\hline$C_{1}^{*}$ & $\frac{3}{2}^{+}$ & 0 & $\frac{3}{2}$ & 1 & 2.53 & $1.383\left(Y_{1}^{*}\right)$ \\
\hline$B_{0}^{1}$ & $\frac{1}{2}^{-}$ & 1 & $\frac{1}{2}$ & 0 & 2.81 & 1.67 \\
\hline$B_{1}^{1}$ & $\frac{1}{2}^{-}$ & 1 & $\frac{1}{2}$ & 1 & 2.76 & 1.62 \\
\hline$B_{0}^{* 1}$ & $\frac{1}{2}^{-}$ & 1 & $\frac{1}{2}$ & 0 & 2.55 & 1.41 \\
\hline$B_{1}^{* 1}$ & $\frac{1}{2}^{-}$ & 1 & $\frac{1}{2}$ & 1 & 2.89 & 1.75 \\
\hline$B_{0}^{\prime 1}$ & $\frac{1}{2}^{-}$ & 1 & $\frac{3}{2}$ & 0 & $(2.81)$ & $(1.67)$ \\
\hline$B_{1}^{\prime 1}$ & $\frac{1}{2}^{-}$ & 1 & $\frac{3}{2}$ & 1 & 2.89 & 1.75 \\
\hline$B_{0}^{3}$ & $\frac{3}{2}^{-}$ & 1 & $\frac{1}{2}$ & 0 & 2.83 & 1.69 \\
\hline$B_{1}^{3}$ & $\frac{3}{2}^{-}$ & 1 & $\frac{1}{2}$ & 1 & 2.81 & 1.67 \\
\hline$B_{0}^{* 3}$ & $\frac{3}{2}^{-}$ & 1 & $\frac{1}{2}$ & 0 & 2.65 & 1.52 \\
\hline$B_{1}^{* 3}$ & $\frac{3}{2}^{-}$ & 1 & $\frac{1}{2}$ & 1 & 3.08 & 1.94 \\
\hline$B_{0}^{\prime 3}$ & $\frac{3}{2}^{-}$ & 1 & $\frac{3}{2}$ & 0 & $(2.83)$ & $(1.69)$ \\
\hline$B_{1}^{\prime 3}$ & $\frac{3}{2}^{-}$ & 1 & $\frac{3}{2}$ & 1 & 2.90 & $\cdots$ \\
\hline $\boldsymbol{B}_{0}^{5}$ & $\frac{5}{2}^{-}$ & 1 & $\frac{3}{2}$ & 0 & 2.97 & 1.83 \\
\hline$B_{1}^{\prime 5}$ & $\frac{5}{2}^{-}$ & 1 & $\frac{3}{2}$ & 1 & 2.91 & 1.77 \\
\hline$A_{1}^{* 1}$ & $\frac{1}{2}^{+}$ & 2 & $\frac{3}{2}$ & 1 & 3.23 & $\cdots$ \\
\hline$A_{0}^{3}$ & $\frac{3}{2}^{+}$ & 2 & $\frac{1}{2}$ & 0 & 3.03 & 1.89 \\
\hline$A_{1}^{3}$ & $\frac{3}{2}^{+}$ & 2 & $\frac{1}{2}$ & 1 & 2.98 & 1.84 \\
\hline$A_{1}^{* 3}$ & $\frac{3}{2}^{+}$ & 2 & $\frac{3}{2}$ & 1 & 3.22 & 2.08 \\
\hline$A_{0}^{5}$ & $\frac{5}{2}^{+}$ & 2 & $\frac{1}{2}$ & 0 & 2.96 & 1.82 \\
\hline$A_{1}^{5}$ & $\frac{5}{2}^{+}$ & 2 & $\frac{1}{2}$ & 1 & 3.06 & 1.92 \\
\hline$A_{1}^{* 5}$ & $\frac{5}{2}^{+}$ & 2 & $\frac{3}{2}$ & 1 & 3.21 & 2.07 \\
\hline$A_{1}^{* 7}$ & ${\frac{7^{+}}{2}}^{+}$ & 2 & $\frac{3}{2}$ & 1 & 3.17 & 2.03 \\
\hline
\end{tabular}

handed contributions $\left(\left|f_{+}\right|^{2}+\left|f_{-}\right|^{2}\right)$ obtain contributions from $V V$ and $A A$ terms, whereas the difference $\left(\left|f_{+}\right|^{2}-\left|f_{-}\right|^{2}\right)$ obtains contributions from $V A$ interference terms. From the definition of $u$ and $v$ it is evident that the contribution of the parityviolating $V A$ interference term is down by one power of $E$.

The appropriate asymptotic $q^{2}$ dependence of the helicity form factors will be determined by appealing to the Drell-Yan threshold relation. If one has for $x \rightarrow 1 \nu W_{2} \propto(1-x)^{2 c-1}$ and $\sigma_{S} / \sigma_{T} \propto\left(q^{2}\right)^{-1}$ one finds for the helicity form factors

$$
\begin{aligned}
& f_{ \pm} \propto\left(q^{2}\right)^{-c+1 / 2}, \\
& f_{0} \propto\left(q^{2}\right)^{-c-1 / 2} .
\end{aligned}
$$

On integrating $d \sigma / d q^{2}$ from $q_{\min }^{2}$ to $q_{\max }^{2}$ one has for the integrated cross section $\sigma(E)=\hat{\sigma}_{S}(E)+\hat{\sigma}_{L}(E)$ $+\hat{\sigma}_{R}(E) \equiv \hat{\sigma}_{S}(E)+\hat{\sigma}_{T}(E)$

$$
\hat{\sigma}_{S, T} \sim \text { const }-\mid \text { const } \mid E^{-1}+O\left(E^{-2}\right)
$$

(for $c \geqslant 1$ ).

Using the relations between the helicity and invariant form factors given in Appendix A, one can easily determine the asymptotic $q^{2}$ power of the invariant form factors necessary to ensure the behavior given in Eq. (3.4). For example, for the case $\frac{1}{2}^{+} \rightarrow \frac{3}{2}^{+}$, one would have

$$
\begin{aligned}
& G_{0}, G_{1}, G_{2} \propto\left(q^{2}\right)^{-1-c}, \\
& G_{3} \propto\left(q^{2}\right)^{-2-c} .
\end{aligned}
$$

The dangerous role of the $q^{2}$-dependent momentum factors that can lead to spuriously large cross sections can already be appreciated in this case. If one were to naively choose dipole forms for all the invariant form factors, i.e., $G_{i} \propto\left(q^{2}\right)^{-2}$, one would then have $\nu W_{2} \propto(1-x)^{0}$ and $\sigma_{S} / \sigma_{T} \propto q^{2}$, as well as

$$
\begin{aligned}
& \hat{\sigma}_{S} \propto \ln E, \\
& \hat{\sigma}_{T} \propto \text { const }-\mid \text { const } \mid E^{-1}
\end{aligned}
$$

for the $q^{2}$ integrated cross sections. Apart from the fact that such a choice is not in agreement with the Drell-Yan threshold relation, a logarithmic growth of the total cross section for single-resonance production does not seem to be realistic from what is known in the corresponding electroproduction cases or from quasielastic neutrino scattering, which all show a flat energy depenence.

\section{CURRENT MATRIX ELEMENTS}

Having dealt with the charmed-baryon spectrum and the production kinematics, we shall now turn to the calculation of the weak-current matrix elements (CME's) that appear in the cross-section formula Eq. (3.1) and which constitute the dynamical content of the production process. First we calculate their $q^{2}=0$ values using the quark model as dynamical input.

Current matrix element (CME) at $\boldsymbol{q}^{2}=0$ in the quark model

The internal wave function of baryons composed of three quarks is written in momentum space as follows:

$$
\Phi_{\left(J, J_{z}\right)}(\overrightarrow{\mathrm{p}})=\sum_{S_{z}, L_{z}} C\left(S, S_{z} ; L, L_{z} ; J, J_{z}\right) \int \prod_{i=1}^{3} d^{3} k_{i} \delta^{3}\left(\overrightarrow{\mathrm{p}}-\sum_{i=1}^{3} \overrightarrow{\mathrm{k}}_{i}\right) \phi_{\left(L, L_{z}\right)}\left(\overrightarrow{\mathrm{k}}_{1}, \overrightarrow{\mathrm{k}}_{2}, \overrightarrow{\mathrm{k}}_{3}\right) \chi_{123^{2}}^{\left(S, S_{z}\right)}
$$


where $\phi$ and $\chi$, respectively, represent the internal space and the spin-unitary-spin wave functions, $C\left(S, S_{z} ; L, L_{z} ; J, J_{z}\right)$ is a Clebsch-Gordan coefficient, and $\overrightarrow{\mathbf{k}}_{i}(i=1,2,3)$ is the three-momentum of the $i$ th quark. Note that the spin-unitary-spin wave function can easily be obtained from those of baryons with $C=0$ by the replacement $\lambda \rightarrow c$.

The current matrix elements of charmed-particle production are calculated according to Fig. 2, In the isobar rest frame one has

$$
\begin{aligned}
& \Phi_{F\left(J, J_{z}\right)}\left(\overrightarrow{\mathrm{p}}_{F}\right) J_{\mu}^{V, A}(0) \Phi_{I\left(1 / 2, J_{z}^{\prime}\right)}\left(\overrightarrow{\mathrm{p}}_{i}\right) \\
& =\sum_{S_{z}, L_{z}} C^{*}\left(S, S_{z} ; L, L_{z} ; J, J_{z}\right) \int \prod_{i=1}^{3} d^{3} k_{i} \delta^{3}\left(\overrightarrow{\mathrm{p}}_{F}-\sum_{i=1}^{3} \overrightarrow{\mathrm{k}}_{i}\right) \phi_{F\left(L, L_{z}\right)}^{+}\left(\overrightarrow{\mathrm{k}}_{1}, \overrightarrow{\mathrm{k}}_{2}, \overrightarrow{\mathrm{k}}_{3}\right) \chi_{F, 123}^{+\left(S, S_{z}\right)} \Gamma_{\mu}^{V, A} \chi_{I, 123}^{\left(1 / 2, J_{z}^{\prime}\right)} \phi_{I(0)}\left(\overrightarrow{\mathrm{k}}_{1}, \overrightarrow{\mathrm{k}}_{2}, \overrightarrow{\mathrm{k}}_{3}-\overrightarrow{\mathrm{q}}\right) \\
& =\sum_{L_{z}}\left\langle\mathfrak{e}\left(L, L_{z} ; J, J_{z}\right)\right\rangle \int \prod_{i=1}^{3} d^{3} k_{i} \delta^{3}\left(\overrightarrow{\mathrm{p}}_{F}-\sum_{i=1}^{3} \overrightarrow{\mathrm{k}}_{i}\right) \phi_{F\left(L, L_{z j}{ }^{j}\right.}^{+}\left(\overrightarrow{\mathrm{k}}_{1}, \overrightarrow{\mathrm{k}}_{2}, \overrightarrow{\mathrm{k}}_{3}\right) \phi_{I(0)}\left(\overrightarrow{\mathrm{k}}_{1}, \overrightarrow{\mathrm{k}}_{2}, \overrightarrow{\mathrm{k}}_{3}-\overrightarrow{\mathrm{q}}\right) \vec{u}_{c}\left(\overrightarrow{\mathrm{k}}_{3}\right) \Gamma_{\mu}^{V, A} u_{n}\left(\overrightarrow{\mathrm{k}}_{3}-\overrightarrow{\mathrm{q}}\right),
\end{aligned}
$$

where the relevant charm-changing current operator is $\Gamma_{\mu}^{V}+\Gamma_{\mu}^{A}=\gamma_{\mu}\left(1-i \gamma_{5}\right) \sin \theta_{C}$ with $\sin \theta_{C}=0.235 \pm 0.05$. $\left\langle\mathfrak{e}\left(L, L_{z} ; J, J_{z}\right)\right\rangle$ is a Clebsch-Gordan coefficient factor.

In the nonrelativistic model the $\vec{k}$ dependence of the Dirac spinor is neglected and instead the Pauli spinors are used. Such an approximation is reliable only if $|\vec{q}|$ and the cutoff momentum for the $\vec{k}$ 's in $\phi$ are small. In the present case, however, the mass difference between the $\pi$ and $c$ quarks is so large that $|\overrightarrow{\mathrm{q}}|$ is very large even at $q^{2}=0$. Therefore, one may neglect the $\overrightarrow{\mathrm{k}}$ dependence of the Dirac spinors, but not their $\vec{q}$ dependence. In the above approximation one reduces Eq. (4.2) as follows:

$$
\begin{aligned}
& \sum_{L_{z}}\left\langle\boldsymbol{e}\left(L, L_{z} ; J, J_{z}\right)\right\rangle \bar{u}_{c}(0) \Gamma_{\mu}^{V, A} u_{\Re(}(-\overrightarrow{\mathrm{q}}) \\
& \quad \times \int \prod_{i=1}^{3} d^{3} k_{i} \delta^{3}\left(\overrightarrow{\mathrm{p}}_{F}-\sum_{i=1}^{3} \overrightarrow{\mathrm{k}}_{i}\right) \phi_{F\left(L, L_{z}\right)}^{+}\left(\overrightarrow{\mathrm{k}}_{1}, \overrightarrow{\mathrm{k}}_{2}, \overrightarrow{\mathrm{k}}_{3}\right) \phi_{I(0)}\left(\overrightarrow{\mathrm{k}}_{1}, \overrightarrow{\mathrm{k}}_{2}, \overrightarrow{\mathrm{k}}_{3}-\overrightarrow{\mathrm{q}}\right) .
\end{aligned}
$$

The dependence of the CME on the quark masses $m_{\Re}$ and $m_{c}$ is explicitly contained in the Dirac spinors $u_{\Re}$ and $u_{c}$. The overlap integral in (4.3) is a function of $q^{2}$, which is normalized to 1 at $q^{2}=0$ in the case of the ground-state charmed-baryon $\left(C_{0}, C_{1}, C_{1}^{*}\right)$ production.

The production of excited states is proportional to the overalp integral between the orbital wave functions of the ground state and excited state and is thus proportional to $\left|\vec{q}_{\text {isobar rest system }}\right|^{L} \equiv q_{c}^{L}$. The

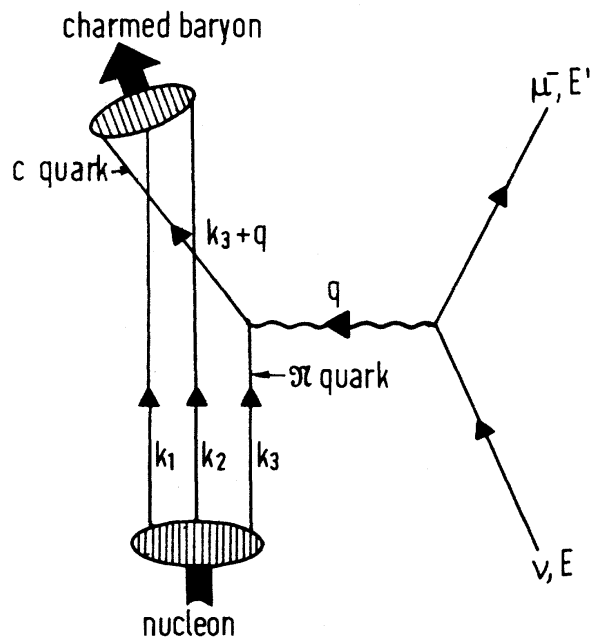

FIG. 2. Quark-model diagram of the neutrino production of charmed baryons. actual value of the overlap is model-dependent but can be calculated once a particular quark model is specified. Here we shall be treating the following two cases:

$$
\text { Model I: } \begin{aligned}
\left.\int \phi_{F}^{+} \phi_{I}\right|_{a^{2}=0}= & {\left[\left(\frac{2}{\Omega}\right)^{1 / 2} q_{c}\right]^{L} } \\
& \times\left.\exp \left[-\frac{m^{2} q_{0 c}}{\Omega\left(M^{2}+m^{2}\right)}\right]\right|_{\alpha^{2}=0},
\end{aligned}
$$

Model II: $\left.\int \phi_{F}^{+} \phi_{I}\right|_{\alpha^{2}=0}=\left.\left(\frac{q_{c}}{M}\right)^{L}\right|_{\alpha^{2}=0}$.

The first case corresponds to the relativistic quark model introduced by Feynman, Kisslinger, and Ravndal, ${ }^{15}$ and the second case is abstracted from the models of Refs. 16 and 17.

In the case of model I, $\Omega$ denotes the spring constant of the harmonic-oscillator potential and is set equal to $\Omega=1.05 \mathrm{GeV}^{2}$ for the noncharmed-particle spectrum. In the case of the $\Delta C=1$ transitions treated here one would possibly have to consider larger values of $\Omega$. This shall be discussed in the next section when we present the numerical results.

In the case of model II, the large value of $q_{c}$ at $q^{2}=0$, i.e., $q_{c}=\left(M^{2}-m^{2}\right) / 2 M$ is scaled to the large mass $M$, and thus one avoids that the orbital overlap increases with the mass of the higher states. The appearance of $M$ as scaling mass is due to a Lorentz-contraction effect of the spatial wave 
function, as can be seen in the following two models. One is the relativistic quark model proposed by Fujimura, Kobayashi, and Namiki (FKN), ${ }^{6}$ and the other is a model proposed by Cocho, Fronsdal, Grodsky, and White (CFGW) ${ }^{17}$ based on the U(3.1) group as a relativistic generalization of the nonrelativistic harmonic oscillator. One obtains the following results:

$$
\begin{aligned}
& \int \phi_{F}^{+} \phi_{I}=C(L)\left[\frac{q_{c}}{(2 \alpha)^{1 / 2} \gamma}\right]^{L} I\left(q^{2}\right)(\mathrm{FKN}), \\
& \int \phi_{F}^{+} \phi_{I}=C^{\prime}(L)\left(\frac{q_{c}}{m \gamma}\right)^{L} I^{\prime}\left(q^{2}\right) \quad(\mathrm{CFGW}),
\end{aligned}
$$

where $2 \alpha \simeq 1 \mathrm{GeV}^{2}$,

$$
\gamma=\left(m^{2}+M^{2}-q^{2}\right) / 2 m M,
$$

and $I$ and $I^{\prime}$ stand for the overlap integral between ground states. The factor $\gamma^{-L}$ is due to the Lorentz contraction of the spatial wave function. At $q^{2}=0 \mathrm{Eq}$. (4.5) reads

$$
\begin{aligned}
& \int \phi_{F}^{+} \phi_{I}=C(L)\left[\frac{m\left(M^{2}-m^{2}\right)}{(2 \alpha)^{1 / 2}\left(M^{2}+m^{2}\right)}\right]^{L} I(0) \quad(\mathrm{FKN}) \\
& \int \phi_{F}^{+} \phi_{I}=C^{\prime}(L)\left(\frac{M^{2}-m^{2}}{M^{2}+m^{2}}\right)^{L} I^{\prime}(0) \quad(\mathrm{CFGW})
\end{aligned}
$$

If we take $I(0)=I^{\prime}(0)=1$ for the normalization of the ground-state wave function, ${ }^{18}$ both expressions Eq. (4.6) behave as constants for large $M$, similar to the behavior of model II. The constant factors $C(L)$ and $C^{\prime}(L)$ in the two models have an $L$ dependence, which is model dependent. For $L=1$ and 2 one obtains

$$
\begin{array}{ll}
L=1, & L=2, \\
\frac{1}{3}, & \frac{1}{2} \sqrt{6}(\mathrm{FKN}), \\
1 / \sqrt{3}, & \frac{1}{6} \text { (CFGW). }{ }^{19}
\end{array}
$$

In the limit of $M \gg m$ the orbital overlap corresponding to model II reduces to

$$
\left(\frac{1}{2}\right)^{L} \quad \text { (model II) . }
$$

For $L=1$ and 2 the above value is quite close to the results of the above two models for $M \gg m$. Using explicit masses for $L=1,2$ excited charm states, one finds that the values for the orbital overlap functions at $q^{2}=0$ are quite close to one another in the three cases.

Returning to Eq. (4.3) one can now explicitly calculate the $q^{2}=0$ values of the current matrix elements using quark-model Clebsch-Gordan factors as specified in Eq. (4.3). The results of this calculation are listed in Appendix B in terms of the $q^{2}=0$ values of the helicity form factors. Note that one has the relations

$$
\begin{aligned}
& \sigma\left(B_{0}^{* 1}\right)=\frac{1}{2} \sigma\left(B_{0}^{1}\right), \\
& \sigma\left(B_{0}^{*^{3}}\right)=\frac{1}{2} \sigma\left(B_{0}^{3}\right), \\
& \sigma\left(B_{1}^{*^{1}}\right)=\frac{1}{2} \sigma\left(B_{1}^{1}\right), \\
& \sigma\left(B_{1}^{*^{3}}\right)=\frac{1}{2} \sigma\left(B_{1}^{3}\right) .
\end{aligned}
$$

These relations should hold exactly when the two masses of the two charmed baryons are equal.

In addition to the relations Eq. (4.7) one has

$$
\left\langle B^{*}(C=1, S=0), \lambda^{*}\left|J_{\mu}^{V, A}\right| B(C=0, S=0), \lambda\right\rangle=0
$$

for the six charmed baryons belonging to the mixed-symmetric representation of $\mathrm{SU}(8)$ with total quark spin $\frac{3}{2}$. This is a typical quark-model selection rule following from the internal quark wave-function structure.

For some applications it is also instructive to refer to the $q^{2}=0$ values of transition form factors that result from our quark-model calculation. These are given in Appendix $C$ for the three cases $C_{0}^{+}, C_{1}^{++}$, and $C_{1}^{*^{++}}$.

\section{Continuation of CME to $q^{2} \neq 0$}

In the quark model the $q^{2}$ dependence of CME is determined by (4.3). The $q^{2}$ dependence of the overlap integral, however, is quite model-dependent. Furthermore, it is well known that naive quark-model calculations of the remaining spin part tend to be unreliable for $q^{2} \neq 0$. For example, for the electroproduction of $D_{13}(1520)$ the simple harmonic-oscillator symmetric-quark-model prediction for the $q^{2}$ dependence of the ratio of the $\frac{1}{2}$ to $\frac{3}{2}$ helicity amplitude does not agree with experiment. ${ }^{20}$ On the other hand, it was shown that the $q^{2} \neq 0$ data of the electroproduction of $N^{*}$ can be accounted for quite well if a generalized-vectordominance-model (GVDM) type $q^{2}$ dependence is assumed for the constraint-free invariant form factors. ${ }^{21}$ We therefore take the quark-model results seriously only at $q^{2}=0$ and continue to $q^{2}<0$ in terms of suitable kinematical singularity- and constraint-free invariant amplitudes for which we shall specify a form-factor behavior in terms of charmed mesons in the timelike region in the spirit of the generalized meson-dominance model (GMDM).

For the invariant form factor we shall make a GMDM ansatz in the form of a product of meson poles as predicted by the dual current model ${ }^{22}$ for leading baryon-resonance excitation. ${ }^{23}$ We write

$$
F\left(q^{2}\right)=\prod_{n=0}^{N(c, J)}\left(1-\frac{q^{2}}{m_{M}^{2}+n \alpha^{\prime-1}}\right)^{-1}
$$

where $m_{M}$ is the lowest meson mass, $\alpha^{\prime}$ is the Regge slope, and the number of poles $N(c, J)$ is 
determined by the desired large $-q^{2}$ behavior as discussed above.

The form factors $G_{i}^{V, A}$ will in general obtain contributions from vector mesons $\left(J^{P C}=1^{--}\right)$, axialvector mesons $\left(1^{++}\right.$and $\left.1^{+-}\right)$, pseudoscalar mesons $\left(0^{-+}\right)$, and scalar mesons $\left(0^{++}\right)$. In the case of charm production, the mesons appearing in Eq. (4.9) have $C=1$. Since little is yet known about the details of the mass spectrum of charmed mesons, we shall use one common set of mass values for the charmed mesons. We take this to be the charmedvector meson $D^{*}$, for which we choose $m_{0 *}=2.0$ GeV. ${ }^{4}$ Similarly one lacks information on the Regge slope $\alpha^{\prime}$ of the $C=1$ mesons which determines the masses of the $D^{*}$ recurrences. We shall consider two representative values:

$$
\alpha^{\prime}=0.5 \mathrm{GeV}^{-2}, \quad \alpha^{\prime}=1.0 \mathrm{GeV}^{-2} .
$$

The latter corresponds to the universal slope as determined by the noncharmed-mass spectrum and the former lies between the universal slope $\alpha^{\prime} \simeq 1$ and the slope $\alpha^{\prime}=0.25 \mathrm{GeV}^{-2}$ of the $\psi, \psi^{\prime}$ sequence [see also Eq. (2.6)]. The number of poles $N(c, J)$ that enter in Eq. (4.9) for each individual case can easily be determined using the required $q^{2}$ dependence of the helicity amplitudes as specified in Eq. (3.4). Thus one has for $G_{0}, G_{1}$, and $G_{2} N=3$, 3 , and 3 in the case of $\frac{1}{2}^{+} \rightarrow \frac{1}{2}^{+}$transitions, if $c$ has the canonical value $c=2$. For $\frac{1}{2}^{+} \rightarrow \frac{3}{2}^{ \pm}$one finds $N=3,3,3,4$ for $G, G_{1}, G_{2}, G_{3}$ if $c=2$, and for $\frac{1}{2}^{+} \rightarrow \frac{5}{2}^{+}$ one finds $N=4,4,4,5$. The general rule is that for $J \geqslant \frac{3}{2}$ excitations every additional spin unit requires one additional pole in order to compensate for the $q^{2}$ dependence of the additional multiplicative momentum factor $q_{c} \sim q^{2}$. We shall always assume canonical form-factor behavior $c=2$ except for $C_{1}^{*^{++}}$excitation $\left(\frac{1}{2}^{+}-\frac{3}{2}^{+}\right)$, where we use $c=3$. The reason is that the transition form factor of the SU(4) partner of the $C_{1}^{*^{++}}$, the $\Delta(1236)$, shows a faster than canonical falloff behavior in electroproduction experiments. ${ }^{24}$ We should mention here that the results of the $q^{2}<0$ multipole analysis of Devenish and $\mathrm{Lyth}^{25}$ can be adequately described by the above choice of form factors. ${ }^{21}$ Continuation to spacelike $q^{2}$ is not unique, but our formulation presented here involves the simplest form factors and works for $N \rightarrow N^{*} .^{21}$

\section{NUMERICAL RESULTS AND DISCUSSION}

The model has four parameters-the mass $M$ of the produced charmed baryon, the ratio $R_{q}$ of $c$ quark mass to $(\mathbb{P}, \mathscr{N})$-quark mass, the charmedmeson slope, and the effective mass $m_{D^{*}}$ of the set of $C=1$ mesons that determine the form factor behavior. As central values for these we use the charmed-baryon mass estimates of Sec. II, $R_{q}$ $\equiv m_{c} / m_{(\odot, \Re)} \simeq \frac{1}{2} m_{\psi} / \frac{1}{3} m_{N} \simeq 1.6 / 0.313=5.11$ as discussed in Sec. II, and $\alpha^{\prime}=0.5 \mathrm{GeV}^{-2}$ and $m_{D^{*}}=2.0$ $\mathrm{GeV}$ as discussed in Sec. IV. We also investigate the effect of varying these parameters around their central values.

Using the central values we first show the results of using either model I or model II described in the last section for the orbital overlap for three representative charmed baryons with $L=0,1$, and 2 in Fig. 3. One has for model I using $\Omega=1.05$

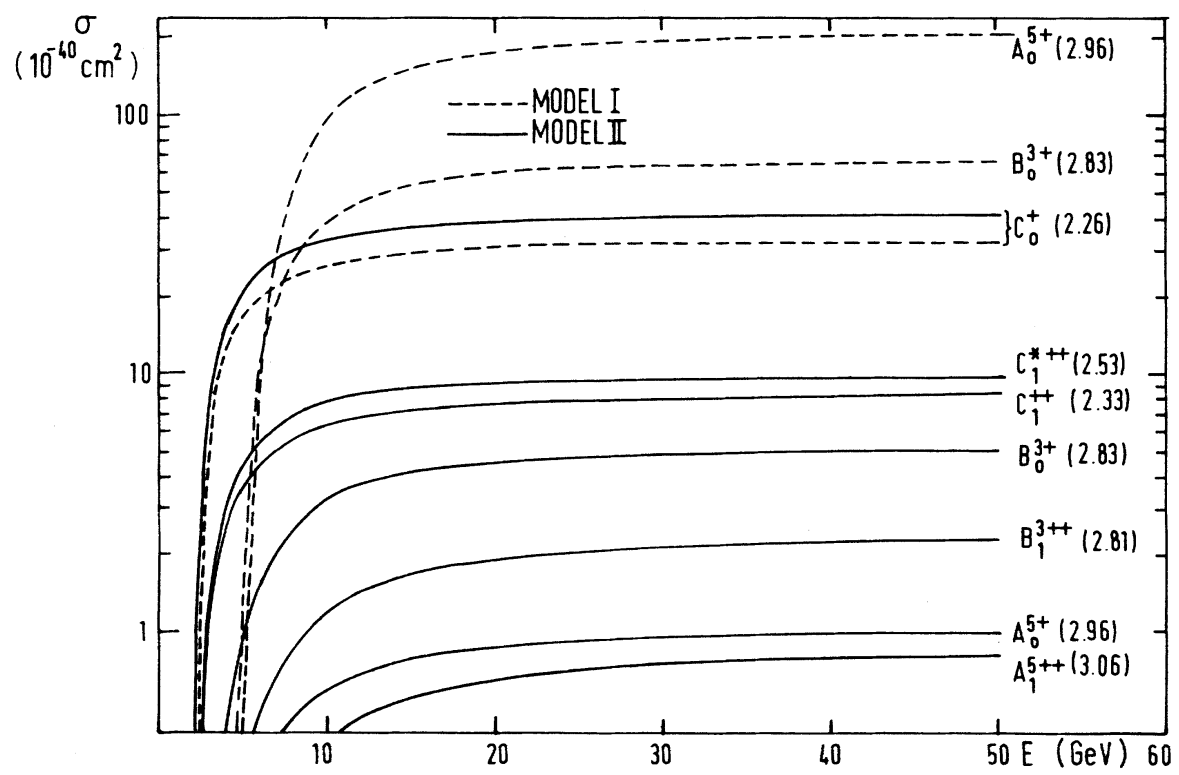

FIG. 3. Production cross sections for seven prominent charmed baryons and resonances using model II (solid lines); production cross sections for $I=0$ states with $J^{P}=\frac{1}{2}^{+}, \frac{3}{2}^{-}, \frac{5}{2}^{+}$using model I (dashed lines). 


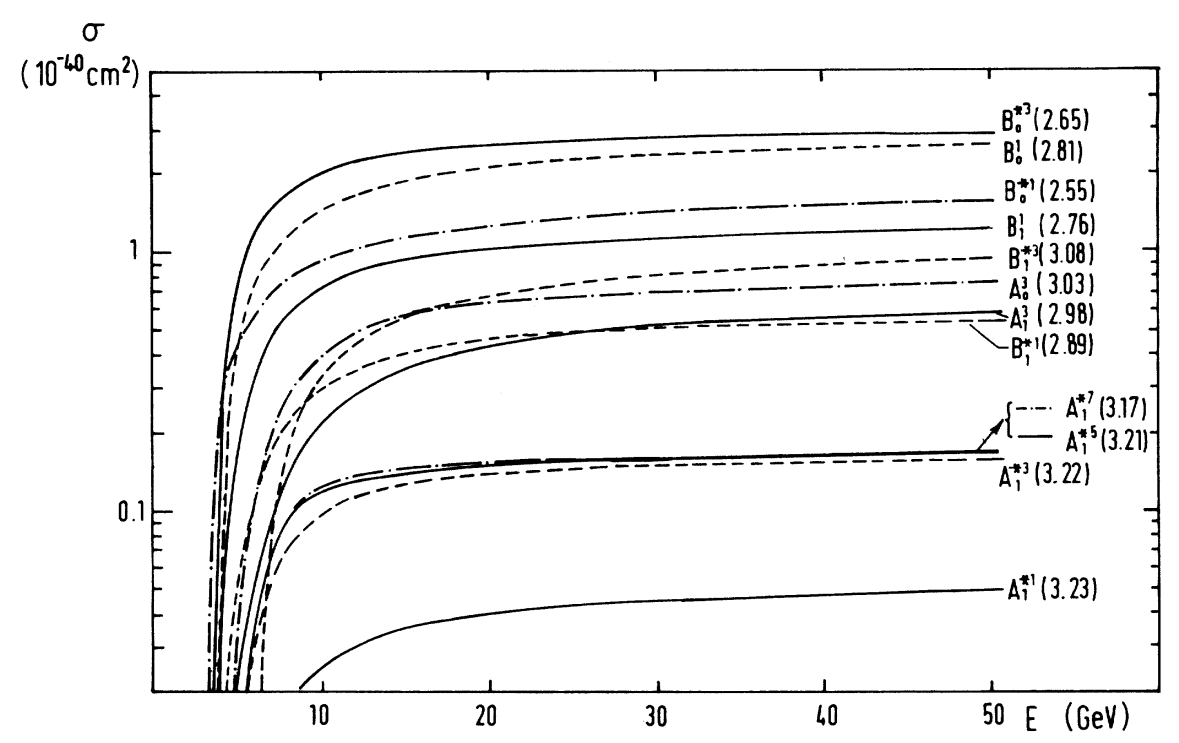

FIG. 4. Production cross sections for 12 charmed-baryon resonances using model II.

$\mathrm{GeV}^{2}$

$\sigma($ ground state $)>\sigma($ excited state $)$,

whereas model II gives

$$
\sigma(\text { ground state })>\sigma(\text { excited state }) \text {. }
$$

If one were to argue that the harmonic-oscillator constant could be larger in cases where charmedparticle production is involved, the behavior, Eq. (5.1), would prevail even for values up to $\Omega=2$ $\mathrm{GeV}^{2}$. That the production of higher $-L$ states should dominate over lower $L$ states is not reasonable in view of the fact that resonance electroproduction shows the opposite behavior, namely that of model II. Henceforth we shall only be using the results of model II.

In Figs. 3 and 4 we show the cross-section results for all of those 19 charmed-baryon states that can be excited, where the cross sections are for proton and neutron targets in the case of $I=1$ and $I=0$ charmed-baryon states, respectively. Among the 19 produced states, the largest four cross sections occur for $C_{0}^{+}, C_{1}^{++}, C_{1}^{*^{++}}$, and $B_{0}^{3+}$. Among the $L=1$ and 2 states the $J^{P}=\frac{3}{2}^{-}$and $\frac{5}{2}^{+}$ states are most prominently produced as in the case in electroproduction experiments. The small deviations from the quark-model sum rules, Eq. (4.6), for the production of $B_{0}^{*^{1}}, B_{0}^{*^{3}}, B_{1}^{*^{1}}$, and $B_{1}^{*^{3}}$ are due to the particle mass differences.

We shall now discuss the effects of varying the input parameters using the four most prominently produced states $C_{0}^{+}, C_{1}^{++}, C_{1}^{*^{++}}$, and $B_{0}^{3}$. In Fig. 5 we vary the effective form-factor parameters $\alpha^{\prime}$ and $m_{D^{*}}$. One has

$$
\sigma\left(\text { small } \alpha^{\prime}\right)>\sigma\left(\text { large } \alpha^{\prime}\right),
$$

$$
\sigma\left(\text { small } m_{D^{*}}\right)<\sigma\left(\text { large } m_{D^{*}}\right),
$$

which can be understood from the explicit form of the form factor, Eq. (4.9). The effects of varying these parameters in the ranges $0.5 \leqslant \alpha^{\prime} \leqslant 1$ and $2.0 \leqslant m_{D^{*}} \leqslant 2.2$ are weak. The dependence on varying the charmed-baryon mass $M$ is given in Table II for $E=50 \mathrm{GeV}$. Again, the results are not very sensitive to mass changes $\Delta M= \pm 0.2 \mathrm{GeV}$. The systematic trend

$$
\sigma(\text { small } M)>\sigma(\text { large } M)
$$

cannot be traced to a single factor.

One can see that using the slightly different mass values of De Rújula, Georgi, and Glashow ${ }^{11}$ for $C_{1}$ and $C_{1}^{*}$ (see Sec. II) has little effect on the crosssection results. For the production of the excited states, e.g. $A_{0}^{5}$, one obtains $\sigma=1.00$ and $\sigma=1.29$ $\times 10^{-40} \mathrm{~cm}^{2}$ at $E=50 \mathrm{GeV}$ using either our mass estimate $M=2.96 \mathrm{GeV}$ or the estimate of Ref. 12, $M=2.69$. This difference is not very significant. From now on we shall always be using the mass estimates of Table I.

In Fig. 6 we show the $R_{q}$ dependence for cross sections at $E=50 \mathrm{GeV}$. For large values of $R_{q}$ one has approximately

$$
\text { ( } E \text { fixed) } \propto R_{\alpha},
$$

which is due to the fact that the helicity amplitudes in our model are proportional to $\left(R_{q}\right)^{1 / 2}$ at large values of $R_{q}$ (see Table IV). ${ }^{26}$ Note that our calculation cannot be extrapolated to the zero-quarkmass limit.

In order to obtain an estimate of the contribution of single-charmed-baryon production to total neutrino cross sections, we have to fold the sum of 


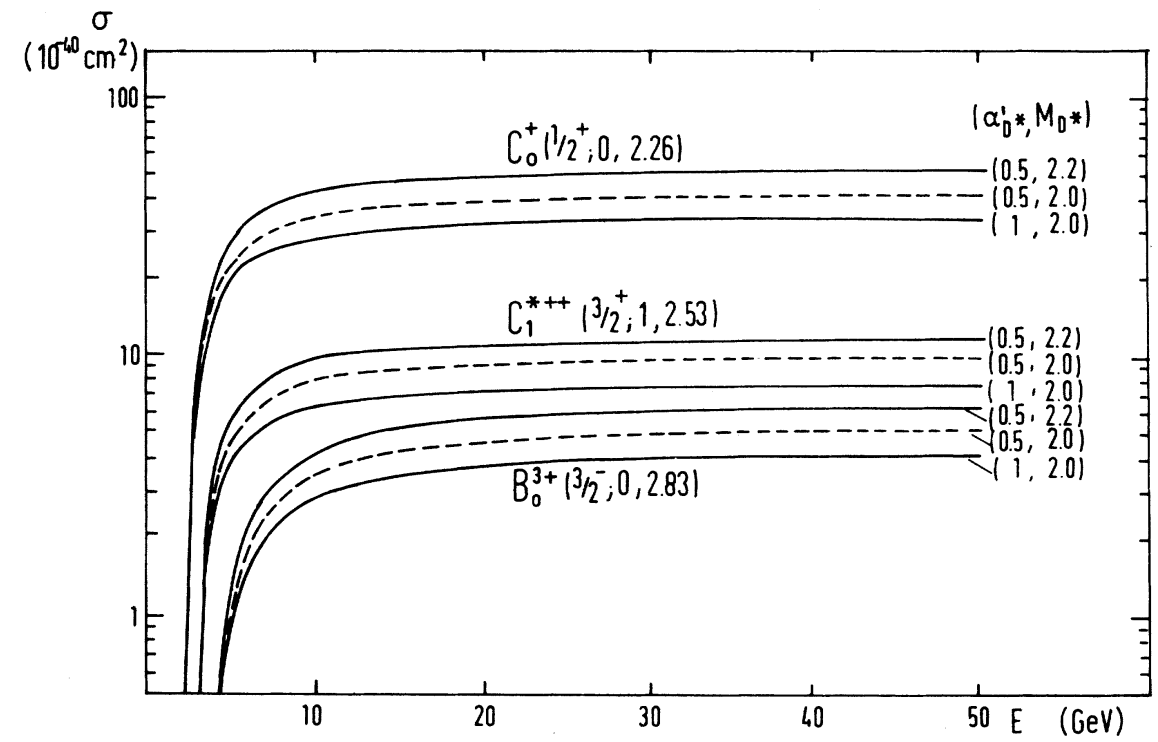

FIG. 5. Dependence of production cross sections on the Regge slope $\alpha^{\prime}$ and $m_{0} *$ (in GeV) for $C_{0}^{+}, C_{1}^{*++}$, and $B^{3+}$.

the 19 cross-section contributions into experimental neutrino fluxes. The fluxes at CERN used by the Gargamelle group and at BNL peak at such low energies $(E \simeq 1.6 \mathrm{GeV})$ that the singlecharmed-baryon production considered here can be expected to be the dominant mechanism of charm production. For the BNL flux we use the parametrization given in Ref. 27. The Gargamelle flux $^{28}$ is parametrized in the following normalized was $(E$ in $\mathrm{GeV})$ :

$\frac{d N_{v}}{d E}=0.20 E\left[(E-1.54)^{2}+0.42 E\right]^{-1}, \quad 0.5 \leqslant E 2.4$,

$\frac{d N_{\nu}}{d E}=0.20 \exp [-0.72(E-2.80)], \quad E>2.4$.

The results are given in Table III. Summing up all 19 contributions to the neutrino scattering on isoscalar nuclear targets, we obtain $\sigma=3.3 \times 10^{-40}$ $\mathrm{cm}^{2}$ for BNL and $\sigma=3.4 \times 10^{-40} \mathrm{~cm}^{2}$ for Gargamelle. This gives $\mathrm{a} \simeq 2 \%$ charm-production rate for these experiments. We have taken

TABLE II. Resonance-mass dependence of the production cross sections for $C_{0}, C_{1}, C_{1}^{*}$, and $B_{0}^{1}$.

\begin{tabular}{|c|c|c|c|}
\hline \multirow{2}{*}{$\begin{array}{l}\text { Resonances } \\
(M(\mathrm{GeV}))\end{array}$} & \multicolumn{3}{|c|}{$\sigma(E=50 \mathrm{GeV})\left(10^{-40} \mathrm{~cm}^{2}\right)$} \\
\hline & $M-0.2$ & $M$ & $M+0.2$ \\
\hline$C_{0}^{+}(2.26)$ & 53 & 41 & 34 \\
\hline$C_{1}^{++}(2.33)$ & 8.8 & 8.4 & 8.1 \\
\hline$C_{1}^{*++}(2.53)$ & 11.5 & 9.7 & 8.4 \\
\hline$B_{0}^{1+}(2.83)$ & 6.0 & 5.1 & 4.6 \\
\hline
\end{tabular}

$$
\sigma_{\text {tot }}^{\nu}\left(\mathrm{cm}^{2}\right)=0.83 \times 10^{-38}(\mathrm{GeV}) .
$$

The single-charmed-baryon production rate rises to $\simeq 4 \%$ with a neutrino beam that peaks at $\simeq 10$ $\mathrm{GeV}$ and is $\simeq 1 \%$ at $50 \mathrm{GeV}$.

In Fig. 7 we show the individual cross sections $\hat{\sigma}_{L}, \hat{\sigma}_{R}$, and $\hat{\sigma}_{S}$ due to left-handed, right-handed, and scalar excitations (see Sec. III) for the two states $C_{0}^{+}$and $C_{1}^{++}$. At most energies except for a region close to threshold $\hat{\sigma}_{s}$ dominates due to the contribution of the nonconserved-current part which has a maximum at $q_{\min }{ }^{2}$. In the case of the

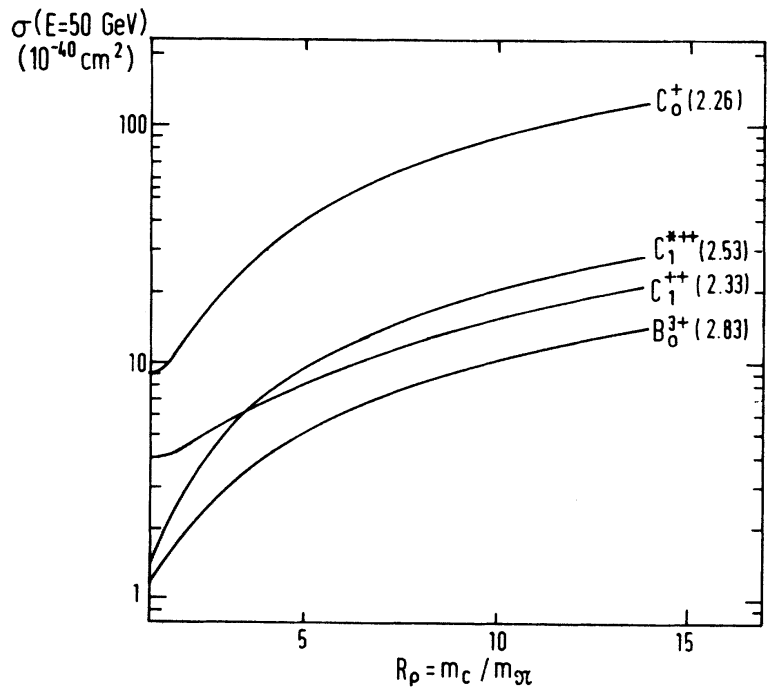

FIG. 6. Dependence of production cross section at $E=50 \mathrm{GeV}$ on the ratio $R_{q}=m_{c} / m(P, \Re)$ for $C_{0}^{+}, C_{1}^{++}$, $C_{1}^{*++}$, and $B_{0}^{3+}$. 
TABLE III. Energy-integrated cross section.

\begin{tabular}{|c|c|c|}
\hline \multirow{2}{*}{$\begin{array}{l}\text { Particles } \\
(M(\mathrm{GeV}))\end{array}$} & \multicolumn{2}{|c|}{$\int d E \times$ flux $(E) \sigma(E)\left(10^{-40} \mathrm{~cm}^{2}\right)$} \\
\hline & Gargamelle & BNL \\
\hline$C_{0}^{+}(2.26)$ & 4.27 & 4.12 \\
\hline$C_{1}^{++}(2.33)$ & 0.68 & 0.65 \\
\hline$C_{1}^{*++}(2.53)$ & 0.67 & 0.64 \\
\hline$B_{0}^{1+}(2.81)$ & 0.062 & 0.066 \\
\hline$B_{1}^{1++}(2.76)$ & 0.025 & 0.028 \\
\hline$B_{0}^{* 1+}(2.55)$ & 0.067 & 0.066 \\
\hline$B_{1}^{* 1++}(2.89)$ & 0.0084 & 0.010 \\
\hline$B_{0}^{3+}(2.83)$ & 0.12 & 0.14 \\
\hline$B_{1}^{3++}(2.81)$ & 0.039 & 0.045 \\
\hline$B_{0}^{* 3+}(2.65)$ & 0.11 & 0.11 \\
\hline$B_{1}^{* 3++}(3.08)$ & 0.0081 & 0.011 \\
\hline$A_{1}^{* 1++}(3.23)$ & 0.0004 & 0.0006 \\
\hline$A_{0}^{3+}(3.03)$ & 0.0085 & 0.011 \\
\hline$A_{1}^{3++}(2.98)$ & 0.0041 & 0.0059 \\
\hline$A_{1}^{* 3}(3.22)$ & 0.0029 & 0.0036 \\
\hline$A_{0}^{5+}(2.96)$ & 0.016 & 0.020 \\
\hline$A_{1}^{5++}(3.06)$ & 0.0063 & 0.0094 \\
\hline$A_{1}^{* 5++}(3.21)$ & 0.0026 & 0.0034 \\
\hline$A_{1}^{* 7++}(3.17)$ & 0.0019 & 0.0026 \\
\hline $\begin{array}{l}\text { Sum over res } \\
\text { nances for } \\
\text { target }\end{array}$ & 3.4 & 3.3 \\
\hline
\end{tabular}

$C_{0}^{+}$the left-handed-current contribution $\hat{\sigma}_{L}$ is almost as strong as $\hat{\sigma}_{S}$ and dominates over the righthanded contribution, whereas for $C_{1}^{++}$both $\hat{\sigma}_{L}$ and $\hat{\sigma}_{R}$ are small and approach each other for larger E.

In Fig. 8 we show differential cross sections $d \sigma / d q^{2}$ for $C_{0}^{+}, C_{1}^{*^{++}}$, and $B_{0}^{3+}$ at two energy values $E=5 \mathrm{GeV}$ and $20 \mathrm{GeV}$. The differential cross section peaks quite strongly at $q^{2}=0$ for $C_{0}^{+}$, whereas the peak is somewhat flatter for the other two cases. Above $E=20 \mathrm{GeV}$ there is practically no change in $d \sigma / d q^{2}$, except, of course, that $q_{\max }^{2}$ becomes larger. From the fact that the dominant production mode at lower energies is $\mathrm{C}_{0}^{+}$one concludes that the production of charmed baryons will center around $q^{2}=0$.

In Fig. 9 we show the effect of renormalizing the axial quark current by a factor $1.23 \times \frac{3}{5}$ for the three cases $C_{0}^{+}, C_{1}^{++}$, and $C_{1}^{*^{++}}$. This change has quite a strong effect on the $C_{1}^{*^{++}}$cross section, which gets reduced approximately by a factor of 0.6 , whereas the reduction in the case of the $C_{0}^{+}$ and $C_{1}^{++}$is not so large. The large reduction of the $C_{1}^{*^{++}}$cross section can be understood from the fact that $C_{1}^{*^{++}}$production is dominated by the axial contribution. The amount of reduction in the other two cases reflects the importance of the axial contribution in each case. In the same figure we also show the effect of enhancing the transverse vector parts of the same three transitions by a factor $(M+m) /\left(m_{(\odot, \Re)}+m_{c}\right)$. Such a possible enhancement is motivated by the observation that relativistic quark models predict magnetic moments

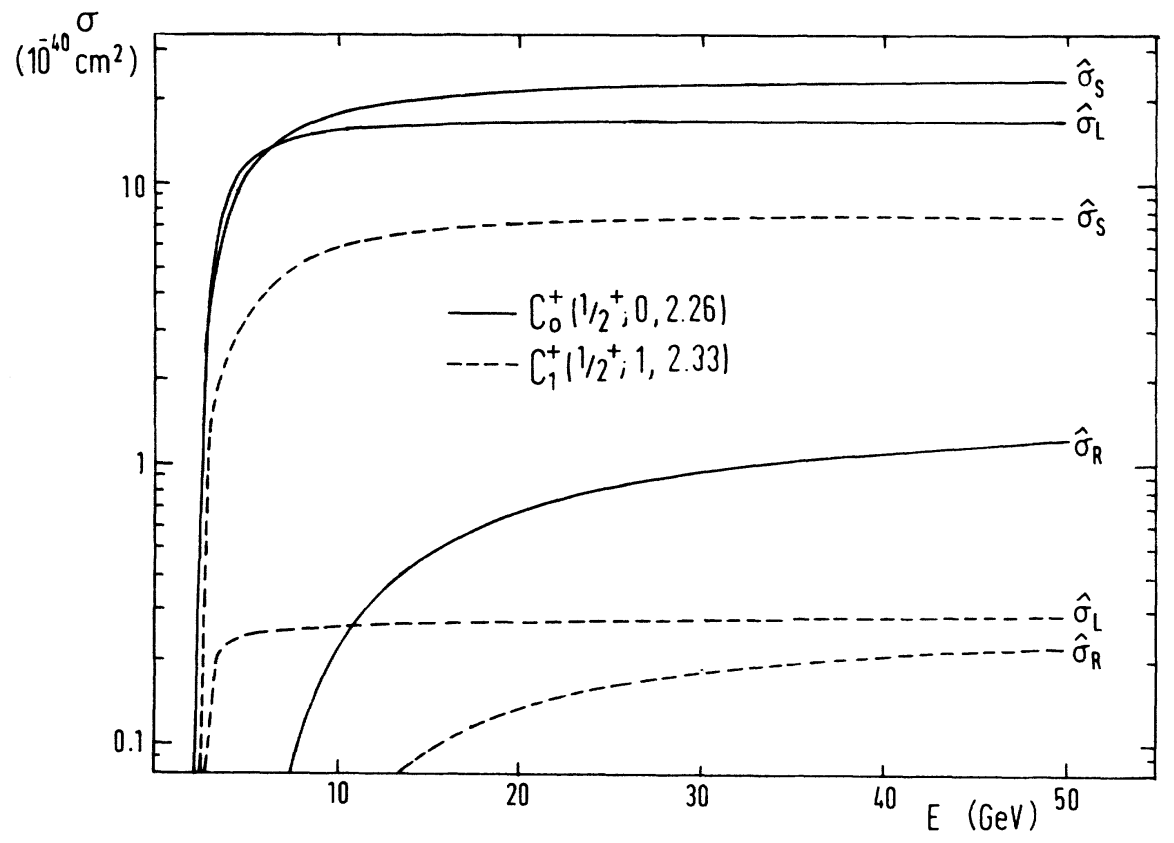

FIG. 7. Scalar, left-handed, and right-handed cross sections $\left(\hat{\sigma}_{S}, \hat{\partial}_{L}\right.$, and $\left.\hat{\partial}_{R}\right)$ for $C_{0}^{+}$and $C_{1}^{++}$. 


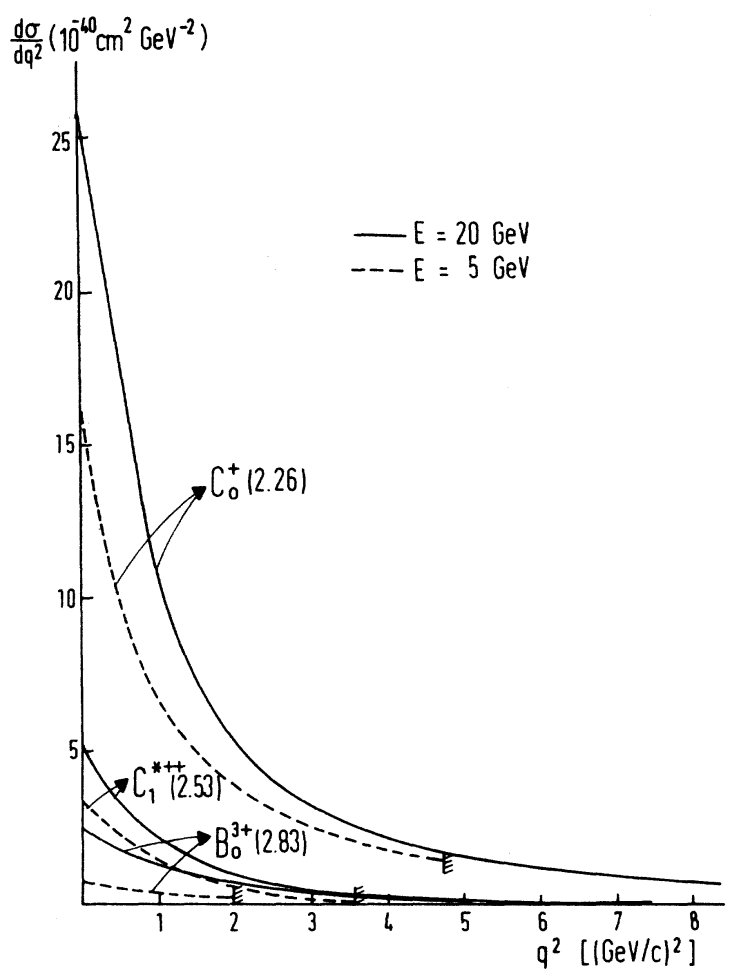

FIG. 8. Differential cross sections for the production of $C_{0}^{+}, C_{1}^{*++}$, and $B^{3+}$ at $E=5 \mathrm{GeV}$ and $20 \mathrm{GeV}$.

and $M 1$ transition moments that are too small by a factor of approximately 3. This is usually remedied by either introducing an anomalous quark coupling or by introducing a mass rescaling factor
$m_{N} / m_{q}$ for the magnetic transitions. With $m_{q}$ $\simeq \frac{1}{3} m_{N}$ or an anomalous quark moment of suitable strength one then obtains the correct magnetic coupling strengths. In the $\Delta C=1$ transition case we replace the magnetic-moment rescaling factor by the corresponding rescaling factor $(M+m)$ / $\left(m_{(\odot, \Re)}+m_{c}\right)$. The strongest effect of this change occurs for $C_{0}^{+}$which is enhanced by a factor $\simeq 1.5$, whereas the effect on $C_{1}^{++}$and $C_{1}^{*^{++}}$is not so strong. It is not clear at present whether the above two modifications of the naive quark modelprediction, which are clearly important in the noncharm sector, are necessary when transitions to heavy charm states are involved.

Finally we discuss the relation of our work with two recent calculations of the neutrino production cross sections of $L=0$ charmed baryons. ${ }^{5,29}$ Finjord and Ravndal's calculation is also based on the quark model; however, their form factors contain the mass parameters of the electromagnetic current instead of the charm mass scale appropriate for the charm-changing current. As remarked already by LS their cross-section estimates are thus unrealistically small. The approach of LS is closer to ours, since they also continue to $q^{2}<0$ using invariant form factors. As already remarked in Ref. 7, the resulting totalcross-section predictions for the $C_{0}^{+}$and the $C_{1}^{++}$ are not very different from ours, indicating that the dominant form factors and their $q^{2}$ behavior close to $q_{\mathrm{min}}{ }^{2}$ are similar in the two models. However, a detailed comparison of the $q^{2}=0$ values of the various form factors reveals that there are

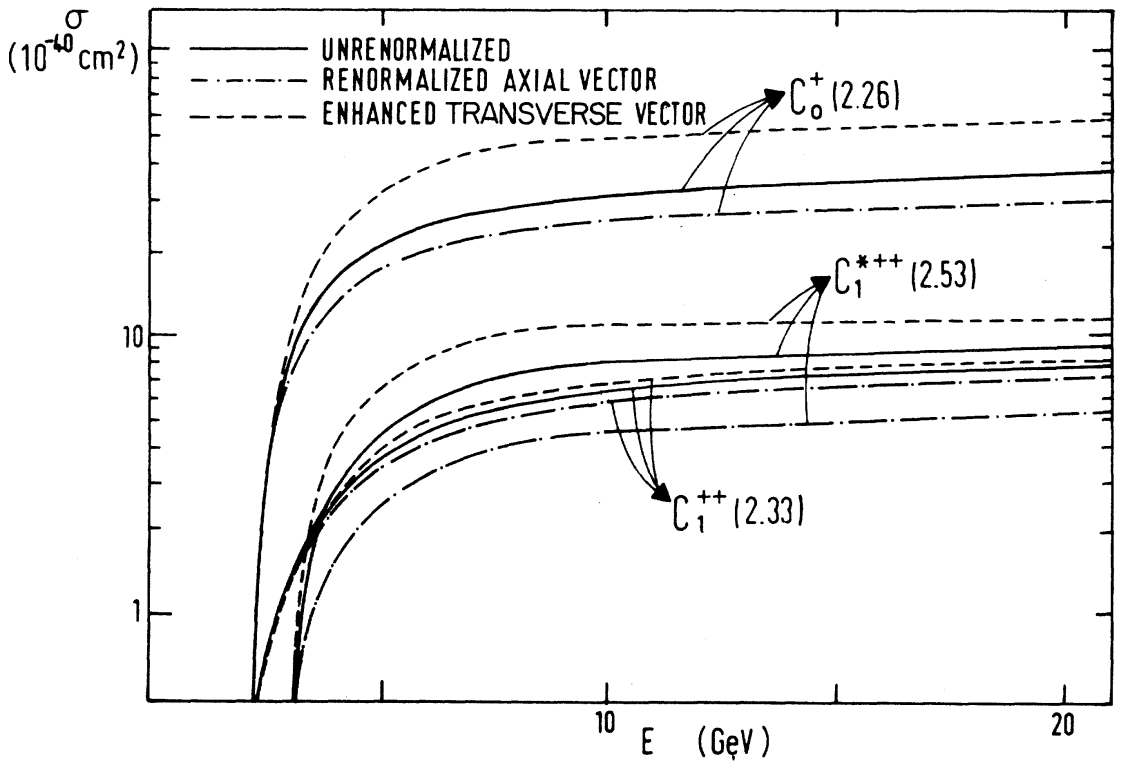

FIG. 9. Production cross sections for $C_{0}^{+}, C_{1}^{++}$, and $C_{1}^{*_{++}}$for the normal case (solid line), renormalized axial-vector current (dash-dot), and enhanced transverse vector part (dashed). 
considerable differences in the two models which would show up in differing polarization predictions. However, such detailed tests will have to be relegated to the distant future. In the case of the $C_{1}^{*^{++}}$production cross section the results of the two calculations are not very different for a region close to the energy threshold (see Ref. 7). However, the form factors of LS do not fall off strongly enough to dampen the effect of the $q^{2}$-dependent momentum factors in the cross-section calculation in this case, and consequently they obtain spuriously large cross-section results for larger energies. As discussed in Sec. III this problem does not occur in our calculation. We do not want to convey the impression that LS presented the isobar-model calculation seriously.

\section{SUMMARY}

We have presented a "hybrid" quark-model calculation of the neutrino production of charmed baryons which uses quark-model results at $q^{2}=0$ and continues these to $q^{2}<0$ via a generalized-meson-dominance continuation. The main results of this calculation can be summarized in the following statements:

1. In the region close to charm-production threshold $(E \simeq 2.5 \mathrm{GeV})$, where single-charmedbaryon production is expected to dominate charm production in neutrino reactions, the $C_{0}^{+}$is produced most copiously, followed by $C_{1}^{*^{++}}$and $C_{1}^{++}$. At a somewhat reduced rate one should also see the production of other conjectured charmedbaryon resonances. Of these the most prominent is $B_{0}^{3+}(2.83)$.

2. Even though single-charmed-baryon production is suppressed by $\sin ^{2} \theta_{c}$, the charm-production rate near threshold can be as large as $\simeq 4 \%$ of the total neutrino cross section.

3. There are a number of parameters in our model calculation that affect the numerical results. Of these, the dependence on the masses of the conjectured charmed-baryon resonances and the dependence on the two form-factor parameters, i.e., the effective meson mass and the trajectory slope, is not very strong. The dependence on the quark mass ratio is stronger and we find approximately

$$
\sigma \propto R_{\alpha}
$$

for $R_{\alpha} \geq 2$.

The calculated cross sections for exclusive charmed-baryon production are large enough to allow one to probe the internal structure of baryons using the charm-changing weak current as a probe. Vice versa, such experiments may be very useful in testing the basic structure of the weak charmchanging current. Our calculation indicates that the cross-section results are quite sensitive to the quark mass ratio. Such a basic symmetry-breaking mechanism could lead to very interesting consequences if there exist more flavored quarks with even heavier masses. Such additional degrees of freedom have been invoked recently as an explanation of the $y$ anomaly in $\bar{\nu}$ reactions and the anomalous behavior of $\sigma(\bar{\nu} p) / \sigma(\nu p)$ above $E=30$ $\mathrm{GeV}$. If new flavored baryons are produced according to the above rule $\sigma \propto R_{\alpha}$, their production cross section could be quite large in $\nu$ and $\bar{\nu}$ interactions, depending of course on the details of the weak-current structure.

\section{ACKNOWLEDGMENT}

The authors would like to express their gratitude for discussions with members of the DESY theory group. Thanks are due to Professor B. Gittelmann for advice on programming. Two of us (C.A. and T.K.) would like to thank Professor H. Joos for his hospitality at DESY. They also thank Professor D. McKay and Dr. M. Krammer for helpful discussions.

Note added. Köpp et al.$^{30}$ discuss the decay of the charmed baryon $C_{0}$ as a test of charm-changing currents. Their production mechanism is identical to that of LS.

\section{APPENDIX A: FORM FACTORS AND HELICITY AMPLITUDES}

For the abnormal-parity transitions $\frac{1}{2}^{+} \rightarrow \frac{3}{2}^{+}, \frac{5}{2}^{-}, \ldots$ we define invariant form factors according to $\left(q=p^{*}-p\right)$

$$
\left\langle B_{J=l+1 / 2}^{*}\left|J_{\mu}(0)\right| B\right\rangle=\frac{G_{C}}{\sqrt{2}} \bar{u}_{B_{1}} \cdots \beta_{l-1} \beta\left(p^{*}\right) q_{B_{1}} \cdots q_{B_{l-1}} \frac{1}{M^{l-1}} \Gamma_{B_{\mu}} i \gamma_{5} u(p)
$$

where

$$
\begin{aligned}
\Gamma_{\beta \mu}= & g_{\beta \mu}\left(G_{0}^{V}-i \gamma_{5} G_{0}^{A}\right)+\frac{1}{M}\left(q_{\beta} \gamma_{\mu}-d g_{B \mu}\right)\left(G_{1}^{V}-i \gamma_{5} G_{1}^{A}\right) \\
& \left.+\frac{1}{M^{2}}\left(q_{\beta} p_{\mu}^{*}-p^{*} q g_{\beta \mu}\right)\left(G_{2}^{V}-i \gamma_{5} G_{2}^{A}\right)+\frac{1}{M^{2}}\left(q_{\beta} q_{\mu}-q^{2} g_{\beta \mu}\right) G_{3}^{V}-i \gamma_{5} G_{3}^{A}\right),
\end{aligned}
$$


and where $G_{C}$ is the weak-coupling constant of Eq. (3.1).

In order to remain close to the treatment of electromagnetic excitation of baryon resonances ${ }^{21}$ we have chosen the latter three of the four independent covariants to be divergence-free, i.e., one has for these $q_{\mu} \Gamma_{B \mu}=0$. The four vector and axial-vector invariants are free of kinematical zeros and constraints (see e.g. Refs. 21 and 31 ).

We define four independent helicity amplitudes (also referred to as helicity form factors) in the isobar rest frame:

$$
\begin{aligned}
& F_{+}=\left\langle\lambda^{*}=\frac{3}{2}\left|\frac{1}{2}\left(J_{x}+i J_{y}\right)\right| \lambda=\frac{1}{2}\right\rangle, \\
& F_{-}=\left\langle\lambda^{*}=-\frac{1}{2}\left|\frac{1}{2}\left(J_{x}-i J_{y}\right)\right| \lambda=\frac{1}{2}\right\rangle, \\
& F_{0}=\left\langle\lambda^{*}=\frac{1}{2}\left|J_{0}\right| \lambda=\frac{1}{2}\right\rangle, \\
& F_{z}=\left\langle\lambda^{*}=\frac{1}{2}\left|J_{z}\right| \lambda=\frac{1}{2}\right\rangle,
\end{aligned}
$$

where $\lambda^{*}$ and $\lambda$ are the helicities of the isobar and the initial baryon. The helicity amplitudes and invariant form factors are related by

$$
\left(\begin{array}{c}
\left(\frac{l}{l+2} \tau_{l+1}\right)^{1 / 2} F_{+}^{V} \\
\left(\tau_{l+1}\right)^{1 / 2} F_{-}^{V} \\
\frac{1}{2}\left(\tau_{l+1}\right)^{1 / 2} F_{z}^{V} / q_{0 c} \\
\frac{1}{2}\left(\tau_{l+1}\right)^{1 / 2} F_{0}^{V} / q_{c}
\end{array}\right)=\frac{1}{\left(Q^{+}\right)^{1 / 2}}\left(\frac{q_{c}}{M}\right)^{2}\left(\begin{array}{cccc}
-M^{2} & M(M+m) & \frac{1}{2}\left(\sigma+q^{2}\right) & q^{2} \\
-M^{2} & M(M+m)-Q^{+} & \frac{1}{2}\left(\sigma+q^{2}\right) & q^{2} \\
-M^{2} / q_{0 c} & M & M & \frac{1}{2 M}\left(\sigma+q^{2}\right) \\
0 & -M & -M & -\frac{1}{2 M}\left(\sigma+q^{2}\right)
\end{array}\right)\left(\begin{array}{c}
G_{0}^{V} \\
G_{1}^{V} \\
G_{2}^{V} \\
G_{3}^{V}
\end{array}\right) .
$$

$M$ and $m$ are the final and initial baryon masses. The inverse of (A3), including the factor in front of the matrix, is given by

$$
\left(Q^{+}\right)^{1 / 2}\left(\frac{M}{q_{c}}\right)^{l} \frac{1}{2 M^{2} q_{c}{ }^{2}}\left(\begin{array}{cccc}
0 & 0 & -2 q_{0 c}{q_{c}}^{2} & -2 q_{0 c} q_{c}{ }^{2} \\
\frac{1}{2} Q^{-} & -\frac{1}{2} Q^{-} & 0 & 0 \\
M(M-m)-Q^{-} & M(M-m) & -2 M q_{0 c}{ }^{2} & -2 M q_{c}{ }^{2} \\
-M^{2} & -M^{2} & 2 M^{2} q_{0 c} & 0
\end{array}\right) .
$$

We have used the abbreviations $Q^{ \pm}=(M \pm m)^{2}-q^{2}$ and $\sigma=M^{2}-m^{2}$. The corresponding relation for the axialvector transition can be obtained from (A3) and (A4) by changing $M \rightarrow-M$ and multiplying by (-1). For the contributions of the three conserved invariants $G_{1}, G_{2}$, and $G_{3}$ one has the current-conservation condition $q_{c} F_{z}+q_{0 c} F_{0}=0 . \quad \tau_{l}$ is the leading power coefficient of the Legendre polynomial $P_{l}(x)$, i.e., $\tau_{l}=$ $(2 l) ! 2^{-l}(l !)^{-2}$.

For the normal-parity series $\frac{1}{2}^{+}-\frac{3}{2}^{-}, \frac{5}{2}^{+}, \ldots$ one defines invariants $G_{i}^{\prime}$ in analogy to Eq. (A2) by multiplying $\Gamma_{B \mu}$ by $i \gamma_{5}$ from the left. The corresponding relation between the helicity amplitudes and form factors can again be obtained from Eq. (A3) by making the substitution $M \rightarrow-M$.

For the transition $\frac{1}{2}^{+} \rightarrow \frac{1}{2}^{+}$, we define invariants by writing

$$
\left\langle B^{*}\left|J_{\mu}(0)\right| B\right\rangle=\frac{G_{C}}{\sqrt{2}} \bar{u}\left(p^{*}\right)\left[\gamma_{\mu}\left(G_{0}^{\prime V}-i \gamma_{5} G_{0}^{\prime A}\right)+\frac{i}{2 M} \sigma_{\mu \nu} q_{\nu}\left(G_{1}^{\prime V}-i \gamma_{5} G_{1}^{\prime A}\right)+\frac{1}{M^{2}}\left(q^{2} \gamma_{\mu}-\not q q_{\mu}\right)\left(G_{2}^{\prime V}-i \gamma_{5} G_{2}^{\prime A}\right)\right] u(p) \text {. }
$$

The three vector and axial-vector invariants are free of kinematical constraints and zeros. In this case one has only three independent helicity amplitudes. Their relation to the form factors is given by [defining $\left.F_{+}=\left\langle\frac{1}{2}\left|\frac{1}{2}\left(J_{x}+i J_{y}\right)\right|-\frac{1}{2}\right\rangle\right]$

$$
\left(\begin{array}{c}
F_{+}^{V} \\
F_{z}^{V} / q_{0 c} \\
F_{0}^{V} / q_{c}
\end{array}\right)=-\left(Q^{-}\right)^{1 / 2} \frac{1}{M^{2}}\left(\begin{array}{ccc}
M^{2} & \frac{1}{2} M(M+m) & q^{2} \\
-\frac{1}{q_{0 c}} M^{2} & -\frac{1}{2} M & -(M+m) \\
-\frac{2 M^{3}}{Q^{-}} & \frac{1}{2} M & (M+m)
\end{array}\right)\left(\begin{array}{c}
G_{0}^{\prime V} \\
G_{1}^{\prime V} \\
G_{2}^{\prime V}
\end{array}\right) \text {. }
$$


TABLE IV. CME at $q^{2}=0$ in the quark model.

\begin{tabular}{lcccccccc}
\hline \hline Particles & $F_{0}^{V}$ & $F_{3}^{V}$ & $F_{+}^{V}$ & $F_{-}^{V}$ & $F_{0}^{A}$ & $F_{3}^{Z}$ & $F_{+}^{A}$ & $F_{-}^{A}$ \\
\hline$C_{0}^{+}$ & $\left(\frac{3}{2}\right)^{1 / 2} Q_{1}$ & $\left(\frac{3}{2}\right)^{1 / 2} Q_{2}$ & $\left(\frac{3}{2}\right)^{1 / 2} Q_{2}$ & $\left(\frac{3}{2}\right)^{1 / 2} Q_{2}$ & $-\left(\frac{3}{2}\right)^{1 / 2} Q_{2}$ & $-\left(\frac{3}{2}\right)^{1 / 2} Q_{1}$ & $\left(\frac{3}{2}\right)^{1 / 2} Q_{1}$ & $-\left(\frac{3}{2}\right)^{1 / 2} Q_{1}$ \\
$C_{1}^{++}$ & $Q_{1}$ & $Q_{2}$ & $-\frac{1}{3} Q_{2}$ & $-\frac{1}{3} Q_{2}$ & $\frac{1}{3} Q_{2}$ & $\frac{1}{3} Q_{1}$ & $-\frac{1}{3} Q_{1}$ & $\frac{1}{3} Q_{1}$ \\
$C_{1}^{*++}$ & 0 & 0 & $\left(\frac{2}{3}\right)^{1 / 2} Q_{2}$ & $-\left(\frac{2}{9}\right)^{1 / 2} Q_{2}$ & $\left(\frac{8}{9}\right)^{1 / 2} Q_{2}$ & $\left(\frac{8}{9}\right)^{1 / 2} Q_{1}$ & $\left(\frac{2}{3}\right)^{1 / 2} Q_{1}$ & $\left(\frac{2}{9}\right)^{1 / 2} Q_{1}$ \\
$B_{0}^{3+}$ & $Q_{1}$ & $Q_{2}$ & 0 & $Q_{2}$ & $-Q_{2}$ & $-Q_{1}$ & 0 & $-Q_{1}$ \\
$B_{1}^{3++}$ & $\left(\frac{2}{3}\right)^{1 / 2} Q_{1}$ & $\left(\frac{2}{3}\right)^{1 / 2} Q_{2}$ & 0 & $-\left(\frac{2}{27}\right)^{1 / 2} Q_{2}$ & $\left(\frac{2}{27}\right)^{1 / 2} Q_{2}$ & $\left(\frac{2}{27}\right)^{1 / 2} Q_{1}$ & 0 & $\left(\frac{2}{27}\right)^{1 / 2} Q_{1}$ \\
$A_{1}^{* 1++}$ & 0 & 0 & $-\left(\frac{2}{9}\right)^{1 / 2} Q_{2}$ & $-\left(\frac{2}{9}\right)^{1 / 2} Q_{2}$ & $\left(\frac{8}{9}\right)^{1 / 2} Q_{2}$ & $\left(\frac{8}{9}\right)^{1 / 2} Q_{1}$ & $-\left(\frac{2}{9}\right)^{1 / 2} Q_{1}$ & $\left(\frac{2}{9}\right)^{1 / 2} Q_{1}$ \\
$A_{0}^{3+}$ & $-\left(\frac{3}{5}\right)^{1 / 2} Q_{1}$ & $-\left(\frac{3}{5}\right)^{1 / 2} Q_{2}$ & 0 & $\left(\frac{3}{5}\right)^{1 / 2} Q_{2}$ & $\left(\frac{3}{5}\right)^{1 / 2} Q_{2}$ & $\left(\frac{3}{5}\right)^{1 / 2} Q_{1}$ & 0 & $-\left(\frac{3}{5}\right)^{1 / 2} Q_{1}$ \\
$A_{0}^{5+}$ & $\left(\frac{9}{10}\right)^{1 / 2} Q_{1}$ & $\left(\frac{9}{10}\right)^{1 / 2} Q_{2}$ & 0 & $\left(\frac{9}{11}\right)^{1 / 2} Q_{2}$ & $-\left(\frac{9}{10}\right)^{1 / 2} Q_{2}$ & $-\left(\frac{9}{10}\right)^{1 / 2} Q_{1}$ & 0 & $-\left(\frac{9}{10}\right)^{1 / 2} Q_{1}$ \\
$A_{1}^{3++}$ & $-\left(\frac{2}{5}\right)^{1 / 2} Q_{1}$ & $-\left(\frac{2}{5}\right)^{1 / 2} Q_{2}$ & 0 & $\left(\frac{2}{45}\right)^{1 / 2} Q_{2}$ & $-\left(\frac{2}{45}\right)^{1 / 2} Q_{2}$ & $-\left(\frac{2}{45}\right)^{1 / 2} Q_{1}$ & 0 & $\left(\frac{2}{45}\right)^{1 / 2} Q_{1}$ \\
$A_{1}^{5++}$ & $\left(\frac{3}{5}\right)^{1 / 2} Q_{1}$ & $\left(\frac{3}{5}\right)^{1 / 2} Q_{2}$ & 0 & $-\left(\frac{1}{15}\right)^{1 / 2} Q_{2}$ & $\left(\frac{1}{15}\right)^{1 / 2} Q_{2}$ & $\left(\frac{1}{15}\right)^{1 / 2} Q_{1}$ & 0 & $\left(\frac{1}{15}\right)^{1 / 2} Q_{1}$ \\
\hline \hline
\end{tabular}

The inverse of $(\mathrm{A} 6)$, including the factor in front of the matrix, is given by

$$
-\frac{2 m}{\left(Q^{-}\right)^{1 / 2} Q^{+}}\left(\begin{array}{ccc}
0 & -\frac{q_{0 c}}{(M-m)} q_{c}{ }^{2} & -\frac{q_{0 c}}{(M-m)} q_{c}{ }^{2} \\
(M+m) & q_{o c}{ }^{2} & q_{c}{ }^{2} \\
-\frac{1}{2} M & -\frac{q_{0 c}}{4(M-m)}\left(3 M^{2}+m^{2}-q^{2}\right) & \frac{M}{2(M-m)} q_{c}{ }^{2}
\end{array}\right) \text {. }
$$

Similar to the case $J>\frac{1}{2}$ the corresponding relations for the axial-vector quantities and for the $\frac{1}{2}^{+} \rightarrow \frac{1}{2}^{-}$ transitions can be obtained from Eq. (A6) by substitution.

\section{APPENDIX B: CME AT $\boldsymbol{q}^{2}=0$ IN THE QUARK MODEL}

We can evaluate the helicity amplitudes at $q^{2}=0$ in the quark model from Eq. (4.3). Evaluations are performed in the isobar rest frame $(\bar{u} u=2 m)$. We obtain the following relations among the helicity amplitudes:

$B_{0}^{1}: \quad F_{i}^{V, A}\left(B_{0}^{1}\right)=-\left(\frac{1}{3}\right)^{1 / 2} \lambda F_{i}^{V, A}\left(C_{0}\right) \quad(i=+, z, 0)$, $F_{-}^{V, A}\left(B_{0}^{1}\right)=\left(\frac{1}{3}\right)^{1 / 2} \lambda F_{-}^{V, A}\left(C_{0}\right)$;

$B_{1}^{1}: F_{i}^{V, A}\left(B_{1}^{1}\right)=-\left(\frac{1}{3}\right)^{1 / 2} \lambda F_{i}^{V, A}\left(C_{1}\right) \quad(i=+, z, 0)$, $F_{-}^{V, A}\left(B_{1}^{1}\right)=\left(\frac{1}{3}\right)^{1 / 2} \lambda F_{-}^{V, A}\left(C_{1}\right) ;$

$B_{0}^{* 1}: F_{i}^{V, A}\left(B_{0}^{* 1}\right)=\left(\frac{1}{2}\right)^{1 / 2} F_{i}^{V, A}\left(B_{0}^{1}\right)$,

$B_{1}^{* 1}: F_{i}^{V, A}\left(B_{1}^{* 1}\right)=\left(\frac{1}{2}\right)^{1 / 2} F_{i}^{V, A}\left(B_{1}^{1}\right)$,

$B_{0}^{*^{3}}: F_{i}^{V, A}\left(B_{0}^{*^{3}}\right)=\left(\frac{1}{2}\right)^{1 / 2} F_{i}^{V, A}\left(B_{0}^{3}\right)$,

$B_{1}^{*^{3}}: F_{i}^{V, A}\left(B_{1}^{*^{3}}\right)=\left(\frac{1}{2}\right)^{1 / 2} F_{i}^{V, A}\left(B_{1}^{3}\right)$;

$$
\begin{aligned}
A_{1}^{* 3}: & F_{i}^{V, A}\left(A_{1}^{* 3}\right)=-\left(\frac{1}{5}\right)^{1 / 2} \lambda^{2} F_{i}^{V, A}\left(C_{1}^{*}\right) \quad(i=-, z, 0), \\
& F_{+}^{V, A}\left(A_{1}^{* 3}\right)=\left(\frac{1}{5}\right)^{1 / 2} \lambda^{2} F_{+}^{V, A}\left(C_{1}^{*}\right), \\
A_{1}^{* 5}: & F_{i}^{V, A}\left(A_{1}^{* 5}\right)=-\left(\frac{3}{35}\right)^{1 / 2} \lambda^{2} F_{i}^{V, A}\left(C_{1}^{*}\right) \quad(i=z, 0), \\
& F_{+}^{V, A}\left(A_{1}^{* 5}\right)=-\left(\frac{18}{35}\right)^{1 / 2} \lambda^{2} F_{+}^{V, A}\left(C_{1}^{*}\right), \\
& F_{-}^{V, A}\left(A_{1}^{* 5}\right)=\left(\frac{3}{35}\right)^{1 / 2} \lambda^{2} F_{-}^{V, A}\left(C_{1}^{*}\right), \\
A_{1}^{* 7}: & F_{i}^{V, A}\left(A_{1}^{* 7}\right)=\left(\frac{18}{35}\right)^{1 / 2} \lambda^{2} F_{i}^{V, A}\left(C_{1}^{*}\right) \quad(i=-, z, 0), \\
& F_{+}^{V, A}\left(A_{1}^{* 7}\right)=\left(\frac{2}{7}\right)^{1 / 2} \lambda^{2} F_{+}^{V, A}\left(C_{1}^{*}\right),
\end{aligned}
$$

where $\lambda=\int \phi_{F}^{*} \phi_{I}$ is defined in Eq. (4.4). The results are given in Table IV, where the contribution of the overlap integral $\lambda^{L}$ is dropped and we define

$$
\begin{aligned}
& Q_{1} \equiv\left(m_{c}+m_{\Re}\right)\left(\frac{m M}{m_{\Re} m_{c}}\right)^{1 / 2}, \\
& Q_{2} \equiv\left(m_{c}-m_{\Re}\right)\left(\frac{m M}{m_{\Re} m_{c}}\right)^{1 / 2} .
\end{aligned}
$$

APPENDIX C: FORM FACTORS AT $q^{2}=0$

The $q^{2}=0$ values of the invariant form factor can be calculated from the relations written down in Appendixes $\mathrm{A}$ and $\mathrm{B}$. We shall give results for transitions to the three ground-state charmed baryons $C_{0}^{+}$, $C_{1}^{++}$, and $C_{1}^{*^{++}}$. 


$$
\begin{aligned}
& C_{1}^{++}: G_{0}^{\prime}(0)=\left(\frac{m m_{c}}{M m_{\Re}}\right)^{1 / 2} \\
& G_{1}^{\prime}(0)=-\frac{1}{M+m}\left[\left(m_{\Re}+m_{c}\right)-\left(m_{c}-m_{\Re}\right) \frac{3 m-M}{3(M-m)}\right]\left(\frac{m M}{m_{\mathfrak{T}} m_{c}}\right)^{1 / 2} \\
& G_{2}^{\prime}{ }^{v}(0)=-\frac{M}{2\left(M^{2}-m^{2}\right)}\left[\left(m_{\Upsilon \tau}+m_{c}\right)-\left(m_{c}-m_{\Upsilon \tau}\right) \frac{7 M^{2}+2 M m+3 m^{2}}{3\left(M^{2}-m^{2}\right)}\right]\left(\frac{m M}{m_{\Upsilon \tau} m_{c}}\right)^{1 / 2}, \\
& G_{0}^{\prime A}(0)=\frac{1}{3}\left(\frac{m m_{c}}{M m_{\Re}}\right)^{1 / 2} \text {, } \\
& G_{1}^{\prime A}(0)=\frac{1}{3(M+m)}\left[\left(m_{\Re}+m_{c}\right)-\left(m_{c}-m_{\Re}\right) \frac{M+m}{M-m}\right]\left(\frac{m M}{m_{\Re} m_{c}}\right)^{1 / 2} \text {, } \\
& G_{2}^{\prime A}(0)=\frac{M}{2(M+m)} G_{1}^{A}(0) \text {. } \\
& C_{0}^{+}: G_{0}^{\prime V}(0)=\left(\frac{3}{2}\right)^{1 / 2}\left(\frac{m m_{c}}{M m_{\Re}}\right)^{1 / 2} \text {, } \\
& G_{1}^{\prime V}(0)=-\left(\frac{3}{2}\right)^{1 / 2} \frac{1}{M+m}\left[\left(m_{\Re}+m_{c}\right)+\left(m_{c}-m_{\Re}\right) \frac{3 M-m}{M-m}\right]\left(\frac{m M}{m_{\Re} m_{c}}\right)^{1 / 2}, \\
& G_{2}^{\prime V}(0)=-\left(\frac{3}{2}\right)^{1 / 2} \frac{M}{2\left(M^{2}-m^{2}\right)}\left[\left(m_{\Re}+m_{c}\right)-\left(m_{c}-m_{\Re}\right) \frac{5 M^{2}-2 M m+m^{2}}{M^{2}-m^{2}}\right]\left(\frac{m M}{m_{\Re} m_{c}}\right)^{1 / 2} \text {, } \\
& G_{0}^{\prime A}(0)=-\left(\frac{3}{2}\right)^{1 / 2}\left(\frac{m m_{c}}{M m_{\mathfrak{N}}}\right)^{1 / 2}, \\
& G_{1}^{\prime A}(0)=-\left(\frac{3}{2}\right)^{1 / 2} \frac{1}{M+m}\left[\left(m_{\Re}+m_{c}\right)-\left(m_{c}-m_{\Re \tau}\right) \frac{M+m}{M-m}\right]\left(\frac{m M}{m_{\Re} m_{c}}\right)^{1 / 2}, \\
& G_{2}^{\prime A}(0)=\frac{M}{2(M+m)} G_{1}^{A}(0) \text {. } \\
& C_{1}^{*^{++}}: G_{0}^{V}(0)=0 \text {, } \\
& G_{1}^{V}(0)=\frac{4}{\sqrt{3}} \frac{\left(m_{c}-m_{\mathfrak{T}}\right) M^{2}}{(M+m)\left(M^{2}-m^{2}\right)}\left(\frac{m M}{m_{\mathfrak{T}} m_{c}}\right)^{1 / 2}, \\
& G_{2}^{V}(0)=-G_{1}^{V}(0) \text {, } \\
& G_{3}^{V}(0)=0 \text {, } \\
& G_{0}^{A}(0)=-\frac{4}{\sqrt{3}} \frac{M}{(M+m)}\left(\frac{m m_{c}}{M m_{\Re}}\right)^{1 / 2}, \\
& G_{1}^{A}(0)=0 \\
& G_{2}^{A}(0)=-\frac{4}{\sqrt{3}}\left(m_{c}-m_{\Re}\right) \frac{M^{2}}{(M+m)\left(M^{2}-m^{2}\right)}\left(\frac{m M}{m_{\Re} m_{c}}\right)^{1 / 2}, \\
& G_{3}^{A}(0)=0 .
\end{aligned}
$$

Note from the cross-section formula Eq. (3.1) that in the forward direction at $q^{2}=0$ only the "nonconserved" invariants $G_{0}$ contribute. The fact that one has nonconserved vector contributions for $C_{0}^{+}$and $C_{1}^{++}$in these $\Delta C=1$ transitions is related to the mass differences of initial and final baryons. The nonvanishing of the nonconserved axial invariants is expected from partial conservation of axial-vector current (PCAC).

*Work supported by the Alexander von Humboldt Foundation.

†Work supported in part by Consejo Nacional de Ciencia y Technologia, Mexico; Present address: Instituto de Fisica, Apdo. Postal 20-364, Mexico 20, D.F. $\ddagger$ Present address: Department of Physics, The University of Tsukubo, Ibaraki, Japan.

${ }^{1}$ B. Knapp et al ., Phys. Rev. Lett. 37,882 (1976).

${ }^{2} \mathrm{~B}$. M. Pontecorvo, in Proceedings of the XVIII International Conference on High Energy Physics, Tbilisi, 
1976, edited by N. N. Bogolubov et al. (JINR, Dubna, 1977), Vol. II, p. B122.

${ }^{3}$ E. G. Cazzoli et al., Phys. Rev. Lett. 34, 1125 (1975).

${ }^{4} \mathrm{G}$. Goldhaber et al., Phys. Rev. Lett. 37, 225 (1976);

I. Perizzi et al ., ibid. 37, 569 (1976); W. Braunschweig et al., Phys. Lett. 63B, 487 (1976); J. Burmester et al ., ibid. 64B, 369 (1976).

${ }^{5}$ R. E. Shrock and B. W. Lee, Phys. Rev. D 13, 2539 (1976).

${ }^{6}$ S. L. Glashow, J. nliopoulos, and L. Maiani, Phys. Rev. D 1,1285 (1970).

${ }^{7}$ Some results of this calculation have already been reported in C. Avilez, T. Kobayashi, and J. G. Körner, Phys. Lett. 66B, 149 (1977).

${ }^{8} \mathrm{H}$. Joos, in Proceedings of the Eleventh Session of the Scottish University Summer School, 1970, edited by J. J. Cumming and H. Osborn (Academic, New York, 1971); T. Tobayashi, Ph.D. thesis, Waseda University, Tokyo, Japan, 1970 (unpublished).

${ }^{9}$ B. W. Lee, C. Quigg, and J. L. Rosner, Phys. Rev. D 15,157 (1977).

${ }^{10}$ Particle Data Group, Rev. Mod. Phys. 48 , S1 (1976).

${ }^{11}$ A. De Rujula, H. Georgi, and S. L. Glashow, Phys. Rev. D 12,147 (1975).

${ }^{12}$ J. Finkelstein and S. F. Tuan, Phys. Rev. D 15,902 (1977).

${ }^{13}$ T. Iwata, T. Takemura, and T. Kobayashi, Prog. Theor. Phys. 42,676 (1969).

${ }^{14}$ F. Ravndal, Nuovo Cimento 18A, 385 (1973).

${ }^{15}$ R. P. Feynman, M. Kislinger, and F. Ravndal, Phys. Rev. D 3, 2706 (1971).

${ }^{16}$ K. Fujimura, T. Kobayashi, and M. Namiki, Prog.

Theor. Phys. 43, 73 (1970); 44, 193 (1970).

${ }^{17} \mathrm{G}$. Cocho, C. Fronsdal, T. T. Grodsky, and R. White, Phys. Rev. 162, 1662 (1969); G. Cocho and J. Flores, Phys. Rev. D 3,157 (1971).

${ }^{18}$ In general $I(0)$ and $I^{\prime}(0)$ can be functions of the masses of initial and final particles.

${ }^{19}$ Taking account of the dipole behavior of the proton magnetic form factor we take one parameter $N$ in the CFGW model (see Ref. 16) as $N=-2$.

${ }^{20}$ F. E. Close and F. J. Gilman, Phys. Lett. 38B, 541 (1972).

${ }^{21}$ R. C. Devenish, T. S. Eisenschitz, and J. G. Körner, Phys. Rev. D 14, 3063 (1976).

${ }^{22} \mathrm{H}$. Sugawara, Tokyo University of Education Report, 1969 (unpublished); I. Ohba, Prog. Theor. Phys. 42, 432 (1969); M. Ademollo and E. del Guidice, Nuovo Cimento 63A, 639 (1969).

${ }^{23} \mathrm{~A}$. Actor, I. Bender, and J. G. Körner, Report No. DESY $75 / 57$ (unpublished).

${ }^{24}$ E. D. Bloom and F. J. Gilman, Phys. Rev. D $\underline{4}, 2901$ (1974).

${ }^{25}$ R. C. Devenish and D. H. Lyth, Nucl. Phys. B93, 109 (1975).

${ }^{26}$ J. C. Pati, A. Salam, and S. Sakakibara proposed a model [Phys. Rev. Lett. 36, 1229 (1976)] in which the anomalous $e \mu$ events seen in $e^{+} e^{-}$annihilation are identified as quark-antiquark events with $(\odot, \mathscr{T})$ - quark masses around $2 \mathrm{GeV}$. If one keeps the mass difference of the $\mathfrak{X}$ and $c$ quarks as $1.287 \mathrm{GeV}$, one obtains $R_{q}=1.6$. For $R_{q} \simeq 1.6$ all production cross sections at high energies are $\frac{1}{3}$ of those for $R_{q}=5.11$.

${ }^{27}$ C. H. Albright, C. Quigg, R. E. Shrock, and J. Smith, Phys. Rev. D 14, 1780 (1977).

${ }^{28} \mathrm{~F}$. W. Bullock, Talk at the Topical Conference on Weak Interactions, Brighton, England, 1976 (unpublished).

${ }^{29}$ J. Finjord and F. Ravndal, Phys. Lett. 58B, 61 (1975).

${ }^{30}$ G. Köpp, L. Seghal, and P. Zerwas, Aachen report, 1976 (unpublished).

${ }^{31}$ W. A. Bardeen and Wu-Ki Tung, Phys. Rev. 173,1423 (1968); R. Tarrach, Nuovo Cimento 28A, 409 (1975). 